Pacific

Journal of

Mathematics

BOUND STATES OF ASYMPTOTICALLY

LINEAR SCHRÖDINGER EQUATIONS

WITH COMPACTLY SUPPORTED POTENTIALS

Mingwen FeI ANd Huicheng Yin 


\title{
BOUND STATES OF ASYMPTOTICALLY LINEAR SCHRÖDINGER EQUATIONS WITH COMPACTLY SUPPORTED POTENTIALS
}

\author{
Mingwen FeI AND Huicheng Yin
}

\begin{abstract}
We study the existence and concentration of bound states to $N$-dimensional nonlinear Schrödinger equation $-\varepsilon^{2} \Delta u_{\varepsilon}+V(x) u_{\varepsilon}=K(x) f\left(u_{\varepsilon}\right)$, where $N \geq 3, \varepsilon>0$ is sufficiently small, and the function $f(s)$ is nonnegative and asymptotically linear at infinity. More concretely, when $f(s) \sim O(s)$ as $s \rightarrow+\infty$, the potential function $V(x)$ lies in $C_{0}^{1}\left(\mathbb{R}^{N}\right)$ with $V(x) \geq 0$ and $V(x) \not \equiv 0$, and $K(x) \geq 0$ is permitted to be unbounded under some other necessary restrictions, we can show that a positive $H^{1}\left(\mathbb{R}^{N}\right)$-solution $u_{\varepsilon}(x)$ exists and concentrates around the local maximum point of the corresponding ground energy function.
\end{abstract}

\section{Introduction and statements of main results}

This paper deals with the problem on the existence and concentration of bound states for the nonlinear Schrödinger equation

$$
\left\{\begin{array}{l}
-\varepsilon^{2} \triangle u_{\varepsilon}+V(x) u_{\varepsilon}=K(x) f\left(u_{\varepsilon}\right), \quad x \in \mathbb{R}^{N}, \\
u_{\varepsilon} \in H^{1}\left(\mathbb{R}^{N}\right), \quad u_{\varepsilon}(x)>0,
\end{array}\right.
$$

where $N \geq 3, \varepsilon>0$ is small, $K(x) \geq 0, V(x) \geq 0$ with $V(x) \not \equiv 0, f(s) \geq 0$ and $f(s) \sim O(s)$ as $s \rightarrow+\infty$, which is asymptotically linear. Such a solution $u_{\varepsilon}$ is called as a bound state for $u_{\varepsilon} \in H^{1}\left(\mathbb{R}^{N}\right)$ and $u_{\varepsilon}(x)>0$.

Consider in particular the superlinear problem given by the equation

$$
\left\{\begin{array}{l}
-\varepsilon^{2} \triangle u_{\varepsilon}+V(x) u_{\varepsilon}=K(x)\left|u_{\varepsilon}\right|^{p-1} u_{\varepsilon}, \quad x \in \mathbb{R}^{N}, \\
u_{\varepsilon} \in H^{1}\left(\mathbb{R}^{N}\right), \quad u_{\varepsilon}>0,
\end{array}\right.
$$

for $N \geq 3$ and $1<p<\frac{N+2}{N-2}$. Under various assumptions on the potential function $V(x) \geq C_{0}>0$ for large $|x|$ or $\lim _{|x| \rightarrow \infty} V(x)=0$ or even $V(x)$ is compactly

This research is supported by NSFC (No. 10931007, No. 11025105), and by the Priority Academic Program Development of Jiangsu Higher Education Institutions. Yin is the corresponding author. MSC2010: 35J10, 35J60, 35Q55, 35J91.

Keywords: nonlinear Schrödinger equation, bound state, asymptotically linear, Harnack inequality, concentration-compactness. 
supported with $V(x) \geq 0$ and $V(x) \not \equiv 0$, the existence of $H^{1}$-positive solutions has been established, and the concentration properties of $u_{\varepsilon}$ can be obtained at a global or local minimum point of the ground energy function $G(\xi) \equiv V^{\theta}(\xi) K^{-2 /(p-2)}(\xi)$ with $\theta=\frac{p}{p-2}-\frac{N}{2}$ (one can see [Ambrosetti et al. 2005; Ambrosetti and Malchiodi 2007; Ambrosetti and Wang 2005; Berestycki and Lions 1983; Bonheure and Van Schaftingen 2008; Byeon and Wang 2006; Dávila et al. 2007; del Pino and Felmer 1996; del Pino et al. 2007; Fei and Yin 2010; Gui 1996; Rabinowitz 1992; Wang and Zeng 1997; Yin and Zhang 2009]).

For the asymptotically linear problem (1-1) with $\varepsilon=1$, there are many papers on the existence of solution in recent years. For examples, in the case of $V(x) \geq C_{0}>0$ for large $|x|$, one can see [Costa and Tehrani 2001; Jeanjean and Tanaka 2002; Liu et al. 2006; Liu and Wang 2004; Stuart and Zhou 1999]; in the special case that $V(x)$ vanishes at infinity like $a /\left(1+|x|^{\sigma}\right) \leq V(x) \leq A$ (the constants $\sigma \in(0,2)$, $a>0$ and $A>0$ ) and some other restrictions, the authors in [Liu et al. 2008] established the existence of bound states.

We now consider the following interesting problems indicated in [Ambrosetti and Malchiodi 2007]: if the potential function $V(x)$ decays faster than $1 /\left(1+|x|^{\sigma}\right)$ with $\sigma \in(0,2)$ at infinity or is compactly supported with $V(x) \geq 0$ and $V(x) \not \equiv 0$, does the bound state of (1-1) still exist? If it exists, what is the concentration profile of $u_{\varepsilon}(x)$ as $\varepsilon \rightarrow 0$ ? In this paper, we will treat these two problems. We only focus on the case that $V(x)$ is compactly supported, since the other cases of $V(x)=O\left(1 /\left(1+|x|^{\sigma}\right)\right)$ with $\sigma \in \mathbb{R}$ can be treated analogously and even more simply.

To proceed, we define the ground energy function $G(\xi)$. The constant coefficient asymptotically linear equation is as follows:

$$
\left\{\begin{array}{l}
-\triangle u(x)+V(\xi) u(x)=K(\xi) f(u), \quad x \in \mathbb{R}^{N}, \\
u \in H^{1}\left(\mathbb{R}^{N}\right), \quad u(x)>0,
\end{array}\right.
$$

where $V(\xi), K(\xi)>0$ with $\xi \in \bar{\Lambda}$, and the meaning of $\Lambda$ is given in assumption $\left(H_{4}\right)$ below.

The associated Euler functional is defined as

$$
I^{\xi}(u)=\frac{1}{2} \int_{\mathbb{R}^{N}}|\nabla u|^{2} d x+\frac{V(\xi)}{2} \int_{\mathbb{R}^{N}}|u|^{2} d x-K(\xi) \int_{\mathbb{R}^{N}} F(u) d x,
$$

where $F(u)=\int_{0}^{u} f(x, \tau) d \tau$.

In the terminology in [Wang and Zeng 1997], the function $G(\xi)=\inf _{u \in \mathcal{M}^{\xi}} I^{\xi}(u)$ is the ground energy function of (1-3) and $\omega(x)$ is a ground state of the functional 
$I^{\xi}$ if $G(\xi)=I^{\xi}(\omega)$, where $M^{\xi}$ is the Nehari manifold, defined as

(1-5) $\mu^{\xi}=$

$$
\left\{u \in H^{1}\left(\mathbb{R}^{N}\right) \backslash\{0\}: \int_{\mathbb{R}^{N}}|\nabla u|^{2} d x+V(\xi) \int_{\mathbb{R}^{N}}|u|^{2} d x=K(\xi) \int_{\mathbb{R}^{N}} f(u) u d x\right\} .
$$

Under certain assumptions, we will solve the constant coefficient asymptotically linear problem (1-3) and prove that the ground state exists and $G(\xi)$ is a continuous function in $\bar{\Lambda}$ in Section 3 below. The assumptions are as follows:

$\left(H_{1}\right) V(x) \in C_{0}^{1}\left(\mathbb{R}^{N}\right), V(x) \geq 0 ; K(x) \in C^{1}\left(\mathbb{R}^{N}\right), K(x) \geq 0$.

$\left(H_{2}\right) f \in C\left(\mathbb{R}, \mathbb{R}^{+}\right) \cap C_{\text {loc }}^{1, \gamma}(\mathbb{R})$ with some constant $\gamma$ satisfying $0<\gamma \leq 1 ; f(s)=0$ for $s \leq 0 ; f(s)=O\left(s^{\alpha}\right)$ with some $\alpha>1$ near $s=0$.

$\left(H_{3}\right) f(s) / s$ is a nondecreasing function for $s>0$ and

$$
\frac{f(s)}{s} \rightarrow l \in(0,+\infty) \text { as } s \rightarrow+\infty \text {. }
$$

$\left(H_{4}\right)$ There exists a smooth bounded domain $\Lambda$ of $\mathbb{R}^{N}$ such that $V(x)>0, K(x)>0$ on $\bar{\Lambda}$ and

$$
\begin{aligned}
\mu^{*} & \equiv \max _{\xi \in \bar{\Lambda}} \frac{V(\xi)}{K(\xi)}<l, \\
0<c_{0} & \equiv \inf _{\xi \in \Lambda} G(\xi)<\inf _{\xi \in \partial \Lambda} G(\xi) .
\end{aligned}
$$

$\left(H_{5}\right)$ Let $N \geq 5$. There exist some constants $k>0$ and $\beta<(\alpha-1)(N-2)-2$ such that

$$
0 \leq K(x) \leq k(1+|x|)^{\beta} \quad \text { in } \mathbb{R}^{N} .
$$

Our main results in this paper can be stated as follows:

Theorem 1.1 (existence and concentration). Let assumptions $\left(H_{1}\right)-\left(H_{5}\right)$ hold.

(i) Equation (1-1) has at least one bound state $u_{\varepsilon}$ provided that $\varepsilon$ is small.

(ii) $u_{\varepsilon}$ has exactly one maximum point $x_{\varepsilon} \in \Lambda$, which satisfies

$$
C_{1} \leq u_{\varepsilon}\left(x_{\varepsilon}\right) \leq C_{2}
$$

and

$$
\operatorname{dist}\left(x_{\varepsilon}, M\right) \rightarrow 0 \quad \text { as } \varepsilon \rightarrow 0,
$$

where $C_{1}, C_{2}$ are positive constants independent of $\varepsilon$, and the set $M$ is defined by $M=\left\{x \in \Lambda: G(x)=c_{0}\right\}$. Moreover, if $M$ only contains a single point $x_{0}$, then $u_{\varepsilon}$ is a single peak solution; more precisely,

$$
u_{\varepsilon}(x)=v\left(\frac{x-x_{\varepsilon}}{\varepsilon}\right)+w_{\varepsilon}(x),
$$


where $w_{\varepsilon}(x) \rightarrow 0$ in $C_{\text {loc }}^{2}\left(\mathbb{R}^{N}\right) \cap L^{\infty}\left(\mathbb{R}^{N}\right)$ as $\varepsilon \rightarrow 0$ and $v \in C^{2}\left(\mathbb{R}^{N}\right) \cap H^{1}\left(\mathbb{R}^{N}\right)$ is the positive solution of the equation

$$
-\triangle v+V\left(x_{0}\right) v=K\left(x_{0}\right) f(v), \quad x \in \mathbb{R}^{N} .
$$

Remark 1.1. In the assumption $\left(H_{5}\right), N \geq 5$ can not be removed to obtain $u_{\varepsilon} \in$ $L^{2}\left(\mathbb{R}^{N}\right)$ in Theorem 1.1 since this is also necessary even for the $N$-dimensional linear Laplacian equation. For more details, one can see Remark 1.2 of [Yin and Zhang 2009]. On the other hand, if we do not require $u_{\varepsilon} \in L^{2}\left(\mathbb{R}^{N}\right)$ in Theorem 1.1, for example, only $u_{\varepsilon} \in L^{q}\left(\mathbb{R}^{N}\right)$ is permitted for some $q>1$, then Theorem 1.1 still holds for all $N \geq 2$ by our proof procedure since $N \geq 5$ is only used in (4-52) of Section 4 to derive $u_{\varepsilon} \in L^{2}\left(\mathbb{R}^{N}\right)$ through the whole paper.

Remark 1.2. In the assumption $\left(H_{2}\right)$, due to $f \in C_{\text {loc }}^{1, \gamma}(\mathbb{R}), f(s)=0$ for $s \leq 0$ and $f(s)=O\left(s^{\alpha}\right)$ near $s=0$ with $\alpha>1$, then we actually have $0<\gamma \leq \min \{1, \alpha-1\}$.

Remark 1.3. With respect to the assumption (1-7) in $\left(H_{4}\right)$, if $V(x) \sim l^{*} /\left(1+|x|^{\beta_{1}}\right)$ with $\beta_{1}>0$ and $K(x) \sim 1 /\left(1+|x|^{\beta_{2}}\right)$ with $0<\beta_{2}<\beta_{1}$ or $V(x) \sim l^{*} e^{-|x|^{\beta_{1}}}$ with $\beta_{1}>0$ and $K(x) \sim e^{-|x|^{\beta_{2}}}$ with $0<\beta_{2}<\beta_{1}$, then for $0<l^{*}<l$, we have $\mu^{*} \leq l^{*}<l$, namely, (1-7) holds true. However, assumption (1-7) does not satisfy the condition $\left(K_{1}\right)$ in [Liu et al. 2008], to the effect that $\sup \left\{\frac{f(s)}{s}: s>0\right\}<\inf \left\{\frac{V(x)}{K(x)}:|x| \geq R_{0}\right\}$ for some $R_{0}>0$, which seems to be crucial to the proof there. On the other hand, the main assumptions $\left(K_{1}\right)$ and (1.8) in Theorem 1.1 of [Liu et al. 2008] are rather restricted. If we use instead of $\left(K_{1}\right)$ the more natural assumption $\sup \left\{\frac{f(s)}{s}: s>0\right\}<$ $\inf \left\{\frac{V(x)}{K(x)}: x \in \mathbb{R}^{n}\right\}$, one can easily derive $l<\inf \left\{\frac{V(x)}{K(x)}: x \in \mathbb{R}^{n}\right\}$ and

$$
\begin{aligned}
\mu^{*} & =\inf f \int_{\mathbb{R}^{N}}\left(|\nabla u|^{2}+V(x) u^{2}\right) d x \int_{\mathbb{R}^{N}} K(x) u^{2} d x \\
& \geq \inf \frac{\int_{\mathbb{R}^{N}}\left(|\nabla u|^{2}+l K(x) u^{2}\right) d x}{\int_{\mathbb{R}^{N}} K(x) u^{2} d x} \geq l,
\end{aligned}
$$

which yields an obvious contradiction between the main assumption $l>\mu^{*}$ of (1.8) and $\left(K_{1}\right)$ in Theorem 1.1 of [Liu et al. 2008].

Remark 1.4. The function $K(x)$ in (1-1) can be permitted to be unbounded if $\alpha>\frac{N}{N-2}$ in view of the assumption (1-9). Moreover, as in Remark 1.2 of [Yin and Zhang 2009], we can illustrate that the restriction on $\beta<(\alpha-1)(N-2)-2$ in (1-9) is optimal in order to obtain the existence of $H^{1}$-positive solution to (1-1).

Remark 1.5. The assumption in $\left(H_{3}\right)$ that $f(s) / s$ is a nondecreasing function for $s>0$ can be removed by more careful analysis than that employed in this paper. This will be done in a forthcoming paper.

Next let's make some comments on the proof of Theorem 1.1. First, we modify the nonlinear term $K(x) f\left(u_{\varepsilon}\right)$ of (1-1) outside $\Lambda$ to $g_{\varepsilon}\left(x, u_{\varepsilon}\right)$, as in [Yin and Zhang 
2009], with the expression

$$
g_{\varepsilon}(x, u)=\min \left\{K(x) f(u), \varepsilon^{3} /\left(1+|x|^{\theta_{0}}\right) u^{+}, \varepsilon /\left(1+|x|^{N}\right)\right\}
$$

for $x \in \mathbb{R}^{N}$ and $u \in \mathbb{R}$, for a positive constant $\theta_{0}$ to be chosen suitably. Then we study the modified equation

$$
-\varepsilon^{2} \Delta u_{\varepsilon}+V(x) u_{\varepsilon}=\chi_{\Lambda}(x) K(x) f\left(u_{\varepsilon}\right)+\left(1-\chi_{\Lambda}(x)\right) g_{\varepsilon}\left(x, u_{\varepsilon}\right)
$$

instead of $-\varepsilon^{2} \triangle u_{\varepsilon}+V(x) u_{\varepsilon}=K(x) f\left(u_{\varepsilon}\right)$ in (1-1). It can be shown that the corresponding Euler functional $I_{\varepsilon}$ of the modified equation is well-defined and has a mountain pass geometry in the weighted Sobolev space

$$
E_{\varepsilon} \equiv\left\{u \in \mathscr{D}^{1,2}\left(\mathbb{R}^{N}\right): \int_{\mathbb{R}^{N}}\left(\varepsilon^{2}|\nabla u|^{2}+V(x)|u|^{2}\right) d x<\infty\right\},
$$

with $\mathscr{D}^{1,2}\left(\mathbb{R}^{N}\right)=\left\{u \in L^{2 N /(N-2)}\left(\mathbb{R}^{N}\right): \nabla u \in L^{2}\left(\mathbb{R}^{N}\right)\right\}$. Motivated by techniques in Chapter IV of [Ekeland 1990] or [Jeanjean and Tanaka 2002], we can use a variant of the mountain pass theorem to find a so-called Cerami sequence, and further show by contradiction that such a Cerami sequence is bounded and prove the existence of a positive solution $u_{\varepsilon}$ to the modified equation.

In order to show such a solution $u_{\varepsilon}$ is just the solution of the original problem (1-1), we require to derive the decay property of solution $u_{\varepsilon}$ and further show $g_{\varepsilon}\left(x, u_{\varepsilon}\right)=K(x) f\left(u_{\varepsilon}\right)$ outside the domain $\Lambda$. To this end, we establish a compactness estimate of integral type to prove that $u_{\varepsilon}$ is small away from their extreme points (see Lemma 4.6 below). Based on such an integral estimate together with the Harnack inequality, we obtain the pointwise decay property of $u_{\varepsilon}$ at infinity and then complete the proof of Theorem 1.1.

Here we point out that some phenomena arising from the asymptotically linear case are quite different from those in superlinear cases, since the exponent $p>1$ of $f(u) \sim u^{p}$ plays a crucial role in showing the concentration-compactness of $u_{\varepsilon}$ and deriving the decay property of $u_{\varepsilon}$ at infinity. (Especially important is the property $F(s) \equiv \int_{0}^{s} f(\tau) d \tau \leq k_{0} f(s) s$, with a positive constant $k_{0}<\frac{1}{2}$ and $s>0$ in superlinear cases; one can see details in [Yin and Zhang 2009; Fei and Yin 2010] and the illustrations before Lemma 4.3 in this paper.) This means that some methods used in [Yin and Zhang 2009] cannot be employed directly here.

Our paper is organized as follows. In Section 2, we replace the nonlinearity $K(x) f\left(u_{\varepsilon}\right)$ outside $\Lambda$ by a suitably truncated function $g_{\varepsilon}\left(x, u_{\varepsilon}\right)$ and give a detailed analysis of the modified equation (1-14), so that the existence of nontrivial positive solution $u_{\varepsilon}$ can be established. In Section 3, we give some preliminary results regarding the properties of the nonlinear Schrödinger equation $-\triangle u+V(\xi) u=$ $K(\xi) f(u)$. In Section 4, we derive an integral decay estimate and use the Harnack inequality to derive the pointwise decay estimate of $u_{\varepsilon}$ at infinity, inspired by 
Lemma 17 of [Ambrosetti et al. 2005] and Lemmas 4.3 and 4.4 of [Yin and Zhang 2009]. From these, together with some involved analysis, we can complete the proof of Theorem 1.1.

We will use the following notations:

$B_{r}$ denotes the ball centered at the origin with the radius $r$.

For a set $A \subset \mathbb{R}^{N}$, we put $A^{\varepsilon}=\left\{\varepsilon^{-1} x: x \in A\right\}$.

\section{Existence of critical points for a modified nonlinear equation}

We define a class of weighted Sobolev spaces as follows:

$$
E_{\varepsilon}:\left\{u \in \mathscr{D}^{1,2}\left(\mathbb{R}^{N}\right): \int_{\mathbb{R}^{N}}\left(\varepsilon^{2}|\nabla u|^{2}+V(x)|u|^{2}\right) d x<\infty\right\}
$$

with $\mathscr{D}^{1,2}\left(\mathbb{R}^{N}\right)=\left\{u \in L^{2 N /(N-2)}\left(\mathbb{R}^{N}\right): \nabla u \in L^{2}\left(\mathbb{R}^{N}\right)\right\}$.

The norm of the space $E_{\varepsilon}$ is denoted by

$$
\|u\|_{\varepsilon}=\left(\int_{\mathbb{R}^{N}}\left(\varepsilon^{2}|\nabla u|^{2}+V(x)|u|^{2}\right) d x\right)^{1 / 2} \quad \text { for } u \in E_{\varepsilon} .
$$

Towards proving Theorem 1.1, it is necessary to modify (1-1) and further discuss the existence of solution to the modified equation.

To this end, we define a function $g_{\varepsilon}(x, \xi)$ by

$$
g_{\varepsilon}(x, \xi)=\min \left\{K(x) f(\xi), \frac{\varepsilon^{3}}{1+|x|^{\theta_{0}}} \xi^{+}, \frac{\varepsilon}{1+|x|^{N}}\right\}, \quad x \in \mathbb{R}^{N}, \xi \in \mathbb{R},
$$

where $\xi^{+}=\max \{\xi, 0\}$, and $\theta_{0}>2$ will be suitably chosen in (4-51).

Set

$$
h_{\varepsilon}(x, \xi)=\chi_{\Lambda}(x) K(x) f(\xi)+\left(1-\chi_{\Lambda}(x)\right) g_{\varepsilon}(x, \xi),
$$

where $\chi_{\Lambda}(x)$ represents the characteristic function of the set $\Lambda$.

We now consider the modified nonlinear equation

$$
-\varepsilon^{2} \Delta u+V(x) u=h_{\varepsilon}(x, u), \quad x \in \mathbb{R}^{N} .
$$

The functional corresponding to (2-1) is

$$
I_{\varepsilon}(u)=\frac{1}{2}\|u\|_{\varepsilon}^{2}-\int_{\Lambda} K(x) F(u) d x-\int_{\mathbb{R}^{N} \backslash \Lambda} G_{\varepsilon}(x, u) d x,
$$

where $F(s)=\int_{0}^{s} f(\tau) d \tau$ and $G_{\varepsilon}(x, s)=\int_{0}^{s} g_{\varepsilon}(x, \tau) d \tau$.

By $\left(H_{2}\right)$ and $\left(H_{3}\right)$, for any $\delta>0$, there exists $C_{\delta}>0$ such that $f(s) \leq \delta s+C_{\delta}|s|^{2^{*}-1}$ 
and further

$$
\int_{\Lambda} K(x) F(u) d x \leq C \delta\|u\|_{\varepsilon}^{2}+C \varepsilon^{-2^{*}}\|u\|_{\varepsilon}^{2^{*}} .
$$

On the other hand, a direct computation yields for $u \in E_{\varepsilon}$

$$
\int_{\mathbb{R}^{N} \backslash \Lambda} G_{\varepsilon}(x, u) d x \leq \int_{\mathbb{R}^{N} \backslash \Lambda} g_{\varepsilon}(x, u) u d x \leq C \varepsilon\|u\|_{\varepsilon}^{2} .
$$

It follows from (2-3) and (2-4) that $I_{\varepsilon}(u)$ is well-defined on $E_{\varepsilon}$. That $I_{\varepsilon}$ lies in $C^{1}\left(E_{\varepsilon}, \mathbb{R}\right)$ is obvious.

Next we show that $I_{\varepsilon}$ has a mountain pass geometry. Given small $\varepsilon>0$, by (2-3) and (2-4), there are two small numbers $\delta$ and $r>0$ such that

$$
I_{\varepsilon}(u) \geq \frac{1}{2}\|u\|_{\varepsilon}^{2}-C \delta\|u\|_{\varepsilon}^{2}-C \varepsilon^{-2^{*}}\|u\|_{\varepsilon}^{2^{*}}-C \varepsilon\|u\|_{\varepsilon}^{2} \geq \frac{1}{4}\|u\|_{\varepsilon}^{2} \quad \text { for }\|u\|_{\varepsilon} \leq r .
$$

We now claim that

$$
\inf _{\psi \in H^{1}\left(\mathbb{R}^{N}\right) \backslash\{0\}} \frac{\int_{\mathbb{R}^{N}}|\nabla \psi|^{2} d x}{\int_{\mathbb{R}^{N}} \psi^{2} d x}=0 .
$$

Indeed, if $\psi_{0}(x) \neq 0 \in H^{1}\left(\mathbb{R}^{N}\right)$, then for any fixed $\lambda \in \mathbb{R}$, one has $\psi_{0}(\lambda x) \in H^{1}\left(\mathbb{R}^{N}\right)$. A direct computation yields that

$$
\int_{\mathbb{R}^{N}}\left|\nabla\left(\psi_{0}(\lambda x)\right)\right|^{2} d x=\lambda^{2-N} \int_{\mathbb{R}^{N}}\left|\nabla \psi_{0}(x)\right|^{2} d x
$$

and

$$
\int_{\mathbb{R}^{N}}\left|\psi_{0}(\lambda x)\right|^{2} d x=\lambda^{-N} \int_{\mathbb{R}^{N}}\left|\psi_{0}(x)\right|^{2} d x .
$$

Therefore, we arrive at

$$
\frac{\int_{\mathbb{R}^{N}}\left|\nabla\left(\psi_{0}(\lambda x)\right)\right|^{2} d x}{\int_{\mathbb{R}^{N}}\left|\psi_{0}(\lambda x)\right|^{2} d x}=\lambda^{2} \frac{\int_{\mathbb{R}^{N}}\left|\nabla \psi_{0}(x)\right|^{2} d x}{\int_{\mathbb{R}^{N}}\left|\psi_{0}(x)\right|^{2} d x} \rightarrow 0 \quad \text { as } \lambda \rightarrow 0,
$$

proving (2-6).

From (2-6), we obtain for any fixed $\xi \in \Lambda$,

$$
\inf _{\psi \in H^{1}\left(\mathbb{R}^{N}\right) \backslash\{0\}} \frac{\int_{\mathbb{R}^{N}}\left(|\nabla \psi|^{2}+V(\xi)|\psi|^{2}\right) d x}{\int_{\mathbb{R}^{N}} K(\xi) \psi^{2} d x}=\frac{V(\xi)}{K(\xi)} .
$$

This, together with (1-7), yields that for fixed $\xi \in \Lambda$ there exists a function $\varphi \in C_{0}^{\infty}\left(\mathbb{R}^{N}\right)$ such that

$$
\frac{\int_{\mathbb{R}^{N}}\left(|\nabla \varphi|^{2}+V(\xi)|\varphi|^{2}\right) d x}{\int_{\mathbb{R}^{N}} K(\xi) \varphi^{2} d x}<l .
$$

Choose $R>0$ such that $B_{R}(\xi) \subset \Lambda$. We define a smooth cut-off function 
$\eta: \mathbb{R}^{+} \rightarrow \mathbb{R}^{+}$satisfying $\eta(t)=1$ if $0 \leq t \leq \frac{R}{4}, \eta(t)=0$ if $t \geq \frac{R}{2}$ and $\left|\eta^{\prime}(t)\right| \leq \frac{8}{R}$.

Set

$$
\varphi_{\varepsilon}(x)=\eta(|x-\xi|) \varphi\left(\frac{x-\xi}{\varepsilon}\right) \in C_{0}^{\infty}(\Lambda) .
$$

Then

$(2-10) \quad I_{\varepsilon}\left(t \varphi_{\varepsilon}\right)$

$$
=\varepsilon^{N}\left(\frac{t^{2}}{2} \int_{\mathbb{R}^{N}}\left(|\nabla \varphi|^{2}+V(\xi)|\varphi|^{2}\right) d x-\frac{1}{2} \int_{\mathbb{R}^{N}} K(\xi) F(t \varphi) d x+o_{\varepsilon}(1)\right) ;
$$

here and below the notation $o_{\varepsilon}(1)$ stands for a quantity which satisfies $o_{\varepsilon}(1) \rightarrow 0$ as $\varepsilon \rightarrow 0$.

Thus we have, for $\varepsilon \leq 1$,

(2-11) $\liminf _{t \rightarrow+\infty} \frac{I_{\varepsilon}\left(t \varphi_{\varepsilon}\right)}{t^{2}} \leq \frac{1}{2} \int_{\mathbb{R}^{2}}\left(|\nabla \varphi|^{2}+V(\xi)|\varphi|^{2}\right) d x-\frac{l}{2} \int_{\mathbb{R}^{N}} K(\xi) \varphi^{2} d x<0$.

Consequently, there exists some $t_{0}>0$ such that $I_{\varepsilon}\left(t_{0} \varphi_{\varepsilon}\right)<0$. This, together with (2-5), means that $I_{\varepsilon}$ has a mountain pass geometry. Let

$$
c_{\varepsilon}=\inf _{\gamma \in \Gamma_{\varepsilon}} \max _{0 \leq t \leq 1} I_{\varepsilon}(\gamma(t)),
$$

where $\Gamma_{\varepsilon}=\left\{\gamma \in C\left([0,1], E_{\varepsilon}\right): \gamma(0)=0, I_{\varepsilon}(\gamma(1))<0\right\}$. By the mountain pass theorem in Chapter IV of [Ekeland 1990], as in [Liu et al. 2008], one has the following lemma.

Lemma 2.1. Under the assumptions $\left(H_{1}\right)-\left(H_{4}\right)$, for small $\varepsilon>0$, there exists a sequence $\left\{u_{n}\right\} \subset E_{\varepsilon}$ such that $I_{\varepsilon}\left(u_{n}\right) \rightarrow c_{\varepsilon}$ and $\left\|I_{\varepsilon}^{\prime}\left(u_{n}\right)\right\|_{E_{\varepsilon}^{\prime}}\left(1+\left\|u_{n}\right\|_{\varepsilon}\right) \rightarrow 0$ as $n \rightarrow \infty$, where $E_{\varepsilon}^{\prime}$ and $\left\|I_{\varepsilon}^{\prime}\left(u_{n}\right)\right\|_{E_{\varepsilon}^{\prime}}$ denote by the dual space of $E_{\varepsilon}$ and the norm of $I_{\varepsilon}^{\prime}\left(u_{n}\right)$ in $E_{\varepsilon}^{\prime}$.

Such a sequence is called a Cerami sequence. Next we will prove the sequence $\left\{u_{n}\right\}$ is bounded in $E_{\varepsilon}$. We reason by contradiction: we assume up to a subsequence that $\left\|u_{n}\right\|_{\varepsilon} \rightarrow+\infty$ as $n \rightarrow+\infty$, and derive a contradiction in Lemmas 2.2 and 2.3.

So assume $\left\|u_{n}\right\|_{\varepsilon} \rightarrow \infty$ and set $\omega_{n}=u_{n} /\left\|u_{n}\right\|_{\varepsilon}$. By the boundedness of $\left\{\omega_{n}\right\}$ in $E_{\varepsilon}$ there exists $\omega \in E_{\varepsilon}$ satisfying, after passing to a subsequence if necessary,

$$
\begin{array}{ll}
\omega_{n} \rightarrow \omega & \text { weakly in } E_{\varepsilon}, \\
\omega_{n} \rightarrow \omega & \text { strongly in } L_{\text {loc }}^{t}\left(\mathbb{R}^{N}\right) \text { with } 2 \leq t<\frac{2 N}{N-2}, \\
\omega_{n} \rightarrow \omega & \text { almost everywhere in } \mathbb{R}^{N} .
\end{array}
$$

Lemma 2.2. Under the assumptions $\left(H_{1}\right)-\left(H_{3}\right)$, if $\left\|u_{n}\right\|_{\varepsilon} \rightarrow+\infty$, then $\omega(x) \geq 0$ with $\omega(x) \not \equiv 0$ and $\omega$ solves the following equation weakly in $E_{\varepsilon}$ :

$$
-\varepsilon^{2} \triangle u+V(x) u=\chi_{\Lambda}(x) l K(x) u .
$$


Proof. Since it follows from Lemma 2.1 that $I_{\varepsilon}^{\prime}\left(u_{n}\right) u_{n}^{-}=o_{n}(1)$, then $\left\|u_{n}^{-}\right\|_{\varepsilon}=o_{n}(1)$ holds true. This means $\left\|\omega_{n}^{-}\right\|_{\varepsilon}=o_{n}(1)$; hence $\omega^{-}=0$ and $\omega \geq 0$.

On the other hand, by Lemma 2.1 and (2-4), we have

$$
\begin{aligned}
o_{n}(1)=\frac{I_{\varepsilon}^{\prime}\left(u_{n}\right) u_{n}}{\left\|u_{n}\right\|_{\varepsilon}^{2}} & =1-\int_{\Lambda} K(x) \frac{f\left(u_{n}\right)}{u_{n}} \omega_{n}^{2} d x-\int_{\mathbb{R}^{N} \backslash \Lambda} \frac{g_{\varepsilon}\left(x, u_{n}\right) u_{n}}{\left\|u_{n}\right\|_{\varepsilon}^{2}} d x \\
& \geq 1-\int_{\Lambda} K(x) \frac{f\left(u_{n}\right)}{u_{n}} \omega_{n}^{2} d x-C \varepsilon ;
\end{aligned}
$$

here and below $o_{n}(1)$ denotes a quantity that vanishes as $n \rightarrow \infty$.

From this, for small $\varepsilon$ and large $n$ we obtain

$$
C \int_{\Lambda} \omega_{n}^{2} d x \geq \int_{\Lambda} K(x) \frac{f\left(u_{n}\right)}{u_{n}} \omega_{n}^{2} d x \geq 1-o_{n}(1)-C \varepsilon \geq \frac{1}{2} .
$$

Combining (2-13) with (2-16) yields $\int_{\Lambda} \omega^{2} d x \geq C$, which obviously leads to $\omega \not \equiv 0$.

Next we prove that $\omega$ satisfies (2-15).

In fact, for any $\phi \in C_{0}^{\infty}\left(\mathbb{R}^{N}\right)$, we have $\frac{I_{\varepsilon}^{\prime}\left(u_{n}\right) \phi}{\left\|u_{n}\right\|_{\varepsilon}}=o_{n}(1)$, which is equivalent to

$$
\begin{aligned}
\int_{\mathbb{R}^{N}}\left(\varepsilon^{2} \nabla \omega_{n} \nabla \phi\right. & \left.+V(x) \omega_{n} \phi\right) d x \\
& =\int_{\Lambda} K(x) \frac{f\left(u_{n}\right)}{u_{n}} \omega_{n} \phi d x-\int_{\mathbb{R}^{N} \backslash \Lambda} \frac{g_{\varepsilon}\left(x, u_{n}\right)}{\left\|u_{n}\right\|_{\varepsilon}} \phi d x+o_{n}(1) .
\end{aligned}
$$

Due to (2-12) and (2-17), there holds

$$
\begin{aligned}
\int_{\mathbb{R}^{N}}\left(\varepsilon^{2} \nabla \omega \nabla \phi\right. & +V(x) \omega \phi) d x \\
& =\lim _{n \rightarrow \infty}\left(\int_{\Lambda} K(x) \frac{f\left(u_{n}\right)}{u_{n}} \omega_{n} \phi d x-\int_{\mathbb{R}^{N} \backslash \Lambda} \frac{g_{\varepsilon}\left(x, u_{n}\right)}{\left\|u_{n}\right\|_{\varepsilon}} \phi d x\right) .
\end{aligned}
$$

Noting that

$$
\int_{\Lambda}\left(K(x) \frac{f\left(u_{n}\right)}{u_{n}} \omega_{n}\right)^{2} d x \leq C \int_{\Lambda} V(x) \omega_{n}^{2} d x \leq C
$$

and

$$
K(x) \frac{f\left(u_{n}\right)}{u_{n}} \omega_{n} \rightarrow l K(x) \omega \text { almost everywhere in } \Lambda,
$$

we get

$$
\lim _{n \rightarrow \infty} \int_{\Lambda} K(x) \frac{f\left(u_{n}\right)}{u_{n}} \omega_{n} \phi d x=\int_{\Lambda} l K(x) \omega \phi d x
$$


In addition, one has

$$
\lim _{n \rightarrow \infty} \int_{\mathbb{R}^{N} \backslash \Lambda} \frac{g\left(x, u_{n}\right)}{\left\|u_{n}\right\|_{\varepsilon}} \phi d x=0 .
$$

Substituting (2-19) and (2-20) into (2-18) yields the conclusion of Lemma 2.2.

Lemma 2.3. Under the assumptions $\left(H_{1}\right)-\left(H_{4}\right)$, Equation (2-15) has no nontrivial solution $\omega(x)$ with $\omega(x) \geq 0$.

Proof. By (1-7), along the proof line of (2-9), there exists $v_{\varepsilon} \in C_{0}^{\infty}(\Lambda)$ such that

$$
\frac{\int_{\Lambda}\left(\varepsilon^{2}\left|\nabla v_{\varepsilon}\right|^{2}+V(x)\left|v_{\varepsilon}\right|^{2}\right) d x}{\int_{\Lambda} K(x) v_{\varepsilon}^{2} d x}<l .
$$

Let $\Lambda_{0}$ be a set satisfying supp $v_{\varepsilon} \subsetneq \Lambda_{0} \subsetneq \Lambda$ and

$$
\mu_{0}=\inf _{\varphi \in C_{0}^{\infty}\left(\Lambda_{0}\right)} \frac{\int_{\Lambda_{0}}\left(\varepsilon^{2}|\nabla \varphi|^{2}+V(x)|\varphi|^{2}\right) d x}{\int_{\Lambda_{0}} K(x) \varphi^{2} d x} ;
$$

then $\mu_{0}<l$.

Due to the compactness of the embedding $H_{0}^{1}\left(\Lambda_{0}\right) \hookrightarrow L^{2}\left(\Lambda_{0}\right)$, a direct argument then shows there exists a nontrivial nonnegative function $v_{0} \in H_{0}^{1}\left(\Lambda_{0}\right)$ such that

$$
-\varepsilon^{2} \triangle v_{0}+V(x) v_{0}=\mu_{0} K(x) v_{0}, \quad x \in \Lambda_{0} .
$$

In addition, by the strong maximum principle [Gilbarg and Trudinger 1983, Lemma 3.4 and Theorem 3.5], one has

$$
v_{0}>0, \quad x \in \Lambda_{0}, \quad \frac{\partial v_{0}}{\partial v}<0, \quad x \in \partial \Lambda_{0} .
$$

Moreover, we can assert that if $\omega \geq 0$ is a nontrivial solution of (2-15), then $\omega \not \equiv 0$ in $\Lambda$ for small $\varepsilon$. Indeed, if $\omega \equiv 0$ in $\Lambda$, we get $\|\omega\|_{\varepsilon}^{2}=0$ by (2-15), which yields a contradiction since $\omega$ is nontrivial.

Hence, we can choose the domain $\Lambda_{0}$ so that $\int_{\Lambda_{0}} K(x) v_{0} \omega d x>0$. In this case, we have

$$
\begin{aligned}
\mu_{0} \int_{\Lambda_{0}} K(x) v_{0} \omega d x & =\int_{\Lambda_{0}}\left(-\varepsilon^{2} \triangle v_{0}+V(x) v_{0}\right) \omega d x \\
& =l \int_{\Lambda_{0}} K(x) v_{0} \omega d x-\int_{\partial \Lambda_{0}} \varepsilon^{2} \frac{\partial v_{0}}{\partial v} \omega d \sigma \geq l \int_{\Lambda_{0}} K(x) v_{0} \omega d x .
\end{aligned}
$$

This means $\mu_{0} \geq l$, which contradicts with $\mu_{0}<l$. Hence we complete the proof of Lemma 2.3.

Combining Lemma 2.2 with Lemma 2.3, we immediately obtain the announced result: 
Lemma 2.4. Under the assumptions $\left(H_{1}\right)-\left(H_{4}\right)$, the sequence $\left\{u_{n}\right\}$ in Lemma 2.1 is bounded in $E_{\varepsilon}$.

Next we state the main result in this section.

Lemma 2.5. Under the assumptions $\left(H_{1}\right)-\left(H_{4}\right)$, for small $\varepsilon>0$, the modified functional $I_{\varepsilon}$ of (2-1) has a nontrivial critical point $u_{\varepsilon} \in E_{\varepsilon}$ with the level $I_{\varepsilon}\left(u_{\varepsilon}\right)=c_{\varepsilon}$. Proof. The boundedness of $\left\{u_{n}\right\}$ in $E_{\varepsilon}$ implies that there exists $u_{\varepsilon} \in E_{\varepsilon}$ satisfying, after passing to a subsequence if necessary,

$$
\begin{array}{ll}
u_{n} \rightarrow u_{\varepsilon} & \text { weakly in } E_{\varepsilon}, \\
u_{n} \rightarrow u_{\varepsilon} & \text { strongly in } L_{\text {loc }}^{t}\left(R^{N}\right) \text { with } 2 \leq t<\frac{2 N}{N-2} .
\end{array}
$$

Next we show $\left\|u_{n}\right\|_{\varepsilon} \rightarrow\left\|u_{\varepsilon}\right\|_{\varepsilon}$ as $n \rightarrow \infty$, which together with (2-22) leads to the strong convergence of $\left\{u_{n}\right\}$ in $E_{\varepsilon}$.

In fact, by $I_{\varepsilon}^{\prime}\left(u_{n}\right) u_{\varepsilon} \rightarrow 0$ and (2-22), we arrive at

$$
\begin{aligned}
o_{n}(1)=\int_{\mathbb{R}^{N}}\left(\varepsilon^{2} \nabla u_{n} \cdot \nabla u_{\varepsilon}\right. & \left.+V(x) u_{n} u_{\varepsilon}\right) d x \\
& -\int_{\Lambda} K(x) f\left(u_{n}\right) u_{\varepsilon} d x-\int_{\mathbb{R}^{N} \backslash \Lambda} g_{\varepsilon}\left(x, u_{n}\right) u_{\varepsilon} d x,
\end{aligned}
$$

which implies

$$
\left\|u_{\varepsilon}\right\|_{\varepsilon}^{2}-\int_{\Lambda} K(x) f\left(u_{n}\right) u_{\varepsilon} d x-\int_{\mathbb{R}^{N} \backslash \Lambda} g_{\varepsilon}\left(x, u_{n}\right) u_{\varepsilon} d x=o_{n}(1) .
$$

In addition, we have

(2-26) $\left\|u_{n}\right\|_{\varepsilon}^{2}-\int_{\Lambda} K(x) f\left(u_{n}\right) u_{n} d x-\int_{\mathbb{R}^{N} \backslash \Lambda} g_{\varepsilon}\left(x, u_{n}\right) u_{n} d x=I_{\varepsilon}^{\prime}\left(u_{n}\right) u_{n}=o_{n}(1)$.

On the other hand, by use of (2-23), we find

$$
\lim _{n \rightarrow \infty} \int_{\Lambda} K(x) f\left(u_{n}\right) u_{n} d x=\lim _{n \rightarrow \infty} \int_{\Lambda} K(x) f\left(u_{n}\right) u_{\varepsilon} d x,
$$

and for any fixed large $R>0$ (without loss of generality, $\Lambda \subset B_{R}$ is assumed),

$$
\lim _{n \rightarrow \infty} \int_{B_{R} \backslash \Lambda} g_{\varepsilon}\left(x, u_{n}\right) u_{n} d x=\lim _{n \rightarrow \infty} \int_{B_{R} \backslash \Lambda} g_{\varepsilon}\left(x, u_{n}\right) u_{\varepsilon} d x .
$$

Thus, in order to obtain $\left\|u_{n}\right\|_{\varepsilon} \rightarrow\left\|u_{0}\right\|_{\varepsilon}$, it follows from (2-25)-(2-28) that we only need to prove the following statement:

For any given $\delta>0$, there exists $R>0$ such that for all $n$

$$
\left|\int_{\mathbb{R}^{N} \backslash B_{R}} g_{\varepsilon}\left(x, u_{n}\right) u_{\varepsilon} d x\right|<\delta, \quad\left|\int_{\mathbb{R}^{N} \backslash B_{R}} g_{\varepsilon}\left(x, u_{n}\right) u_{n} d x\right|<\delta .
$$


It is only enough to check the first inequality in (2-29) since the second one is similar. By direct computations, we have

$$
\left|\int_{\mathbb{R}^{N} \backslash B_{R}} g_{\varepsilon}\left(x, u_{n}\right) u_{\varepsilon} d x\right| \leq \frac{C \varepsilon}{R^{\left(\theta_{0}-2\right) / 2}}\left\|u_{n}\right\|_{\varepsilon}\left\|u_{\varepsilon}\right\|_{\varepsilon} \rightarrow 0 \quad \text { as } R \rightarrow \infty .
$$

The last estimate follows from the choice of $\theta_{0}>2$ and the boundedness of $\left\{u_{n}\right\}$. Thus we have shown that $u_{n} \rightarrow u_{\varepsilon}$ in $E_{\varepsilon}$, which completes the proof of Lemma 2.5.

Remark 2.1. Since $h_{\varepsilon}(x, \xi)$ is Lipschitzian continuous in $\xi$ for fixed $x$, it follows from second order elliptic regularity theory that $u_{\varepsilon}$ is a classical solution of (2-1). Furthermore, $u_{\varepsilon}>0$.

\section{Solving a related constant coefficient problem}

In this section, toward the proof of Theorem 1.1 in Section 4, we study the asymptotically linear problem (1-3) with constant coefficients. Some conclusions and techniques in this section are very similar to those in Section 2, but we give the argument anyway, for the reader's convenience.

We consider the functional $I^{\xi}(u)$ defined in (1-4) for $u \in E \equiv H_{r}^{1}\left(\mathbb{R}^{N}\right)=\{u \in$ $\left.H^{1}\left(\mathbb{R}^{N}\right): u(x)=u(|x|)\right\}$. Set

$$
\|u\|_{\xi}=\left(\int_{\mathbb{R}^{N}}\left(|\nabla u|^{2}+V(\xi)|u|^{2}\right) d x\right)^{1 / 2}
$$

which is a norm equivalent to the $H^{1}\left(\mathbb{R}^{N}\right)$ norm. We now verify that $I^{\xi}$ has a mountain pass geometry. Similar to the proof of (2-5), there are two small numbers $\delta, r>0$ such that

$$
I^{\xi}(u) \geq \frac{1}{2}\|u\|_{\xi}^{2}-C \delta\|u\|_{\xi}^{2}-C\|u\|_{\xi}^{2^{*}} \geq \frac{1}{4}\|u\|_{\xi}^{2} \text { for }\|u\|_{\xi} \leq r .
$$

In addition, by (2-9), there exists a function $\varphi \in H^{1}\left(\mathbb{R}^{N}\right) \backslash\{0\}$ such that

$$
\frac{\int_{\mathbb{R}^{N}}\left(|\nabla \varphi|^{2}+V(\xi)|\varphi|^{2}\right) d x}{\int_{\mathbb{R}^{N}} K(\xi) \varphi^{2} d x}<l .
$$

Let $\varphi^{*}$ be the symmetrization of $\varphi$ (see [Berestycki and Lions 1983, Appen$\operatorname{dix}$ A.III]). Then $\varphi^{*}(x)=\varphi^{*}(|x|)$ is a nonnegative function. Moreover, for any continuous function $H(s)$ such that $H(\varphi(x))$ is integrable in $\mathbb{R}^{N}$ there holds

$$
\int_{\mathbb{R}^{N}} H\left(\varphi^{*}\right) d x=\int_{\mathbb{R}^{N}} H(\varphi) d x
$$

and

$$
\int_{\mathbb{R}^{N}}\left|\nabla \varphi^{*}\right|^{2} d x \leq \int_{\mathbb{R}^{N}}|\nabla \varphi|^{2} d x .
$$


By (3-2)-(3-4), we have

$$
\frac{\int_{\mathbb{R}^{N}}\left(\left|\nabla \varphi^{*}\right|^{2}+V(\xi)\left|\varphi^{*}\right|^{2}\right) d x}{\int_{\mathbb{R}^{N}} K(\xi)\left|\varphi^{*}\right|^{2} d x}<l ;
$$

by the same argument as in (2-11) we can derive

$$
\liminf _{t \rightarrow+\infty} \frac{I^{\xi}\left(t \varphi^{*}\right)}{t^{2}}<0
$$

Thus there exists $t_{0}>0$ such that $I^{\xi}\left(t_{0} \varphi^{*}\right)<0$, showing that $I^{\xi}$ has a mountain pass geometry. Define the mountain level

$$
c_{1}=\inf _{\gamma \in \Gamma} \max _{0 \leq t \leq 1} I^{\xi}(\gamma(t)),
$$

where $\Gamma=\left\{\gamma \in C([0,1], E): \gamma(0)=0, I^{\xi}(\gamma(1))<0\right\}$.

The next two lemmas are established analogously to Lemma 2.1 and Lemma 2.4, respectively.

Lemma 3.1. There exists a sequence $\left\{u_{n}\right\} \subset E$ such that $I^{\xi}\left(u_{n}\right) \rightarrow c_{1}$ and

$$
\left\|\left(I^{\xi}\right)^{\prime}\left(u_{n}\right)\right\|_{H^{-1}}\left(1+\left\|u_{n}\right\|_{\xi}\right) \rightarrow 0 \text { as } n \rightarrow \infty .
$$

Lemma 3.2. The sequence $\left\{u_{n}\right\}$ given in Lemma 3.1 is bounded in E.

Based on Lemma 3.2, we have:

Lemma 3.3. The functional $I^{\xi}$ has a positive critical point $\omega \in H_{r}^{1}\left(\mathbb{R}^{N}\right)$ with the level $I^{\xi}(\omega)=c_{1}$. That is, $\omega$ is a radially symmetric solution to the problem (1-3).

Proof. It follows from the boundedness of $\left\{u_{n}\right\}$ in Lemma 3.2 that there exists $\omega \in E$ satisfying, after passing to a subsequence if necessary,

$$
\begin{array}{ll}
u_{n} \rightarrow \omega & \text { weakly in } E, \\
u_{n} \rightarrow \omega & \text { strongly in } L_{\text {loc }}^{t}\left(R^{N}\right) \text { with } 2 \leq t<\frac{2 N}{N-2} .
\end{array}
$$

As in Lemma 2.5, we only need to show $\left\|u_{n}\right\|_{\xi} \rightarrow\|\omega\|_{\xi}$ as $n \rightarrow \infty$, which together with (3-8) leads to the strong convergence of $\left\{u_{n}\right\}$ in $E$.

Since $\left(I^{\xi}\right)^{\prime}\left(u_{n}\right) \omega \rightarrow 0$ and using (3-8), we arrive at

$$
o_{n}(1)=\int_{\mathbb{R}^{N}}\left(\nabla u_{n} \cdot \nabla \omega+V(\xi) u_{n} \omega\right) d x-\int_{\mathbb{R}^{N}} K(\xi) f\left(u_{n}\right) \omega d x .
$$

This implies

$$
\|\omega\|_{\xi}^{2}-\int_{\mathbb{R}^{N}} K(\xi) f\left(u_{n}\right) \omega d x=o_{n}(1) .
$$


In addition, we have

$$
\left\|u_{n}\right\|_{\xi}^{2}-\int_{\mathbb{R}^{N}} K(\xi) f\left(u_{n}\right) u_{n} d x=o_{n}(1) .
$$

On the other hand, it follows from (3-9) and the Hölder inequality that

$$
\begin{aligned}
\left|\int_{\mathbb{R}^{N}} f\left(u_{n}\right)\left(u_{n}-\omega\right) d x\right| & \leq C \int_{\mathbb{R}^{N}}\left|u_{n}\right|\left|u_{n}-\omega\right| d x \\
& \leq C\left\|u_{n}\right\|_{L^{2}}\left\|u_{n}-\omega\right\|_{L^{2}}=o_{n}(1) .
\end{aligned}
$$

Hence, collecting (3-10)-(3-12) yields $\left\|u_{n}\right\|_{\xi} \rightarrow\|\omega\|_{\xi}$ as $n \rightarrow \infty$ and $I^{\xi}(\omega)=c_{1}$. Moreover, $\omega$ is a nontrivial critical point of $I^{\xi}$ to $E$. By the principle of symmetric criticality (see [Willem 1996, Theorem 1.28]), $\omega$ is also a nontrivial critical point of $I^{\xi}$ to $H^{1}\left(\mathbb{R}^{N}\right)$. In addition, $\omega>0$ can be shown as in Remark 2.1. Therefore, Lemma 3.3 is proved.

Next we assert that the radial function $\omega(x)=\omega(V(\xi), K(\xi) ; x)$ found in Lemma 3.3 is a ground state of the functional $I^{\xi}$, that is,

$$
G(\xi)=I^{\xi}(\omega) .
$$

Obviously, $G(\xi) \leq I^{\xi}(\omega)$ since $\omega \in \mathcal{M}^{\xi}$, $\omega$ being defined in (1-5). What is left is to show $I^{\xi}(\omega) \leq G(\xi)$ in order to get (3-13).

For any $u \in M^{\xi}$, let $u^{*}$ be the symmetrization of $u$. Then $u^{*} \in H^{1}\left(\mathbb{R}^{N}\right)$ and $u^{*} \geq 0$. Consider the function

$$
J(t)=I^{\xi}\left(t u^{*}\right)=\frac{t^{2}}{2} \int_{\mathbb{R}^{N}}\left(\left|\nabla u^{*}\right|^{2}+V(\xi)\left|u^{*}\right|^{2}\right) d x-K(\xi) \int_{\mathbb{R}^{N}} F\left(t u^{*}\right) d x .
$$

A direct computation yields

$$
\begin{aligned}
\lim _{t \rightarrow \infty} \frac{J(t)}{t^{2}} & =\frac{1}{2} \int_{\mathbb{R}^{N}}\left(\left|\nabla u^{*}\right|^{2}+V(\xi)\left|u^{*}\right|^{2}\right) d x-\frac{l K(\xi)}{2} \int_{\mathbb{R}^{N}}\left|u^{*}\right|^{2} d x \\
& \leq \frac{K(\xi)}{2} \int_{\mathbb{R}^{N}}\left(\frac{f\left(u^{*}\right)}{u^{*}}-l\right)\left|u^{*}\right|^{2} d x .
\end{aligned}
$$

In addition, by the Strauss inequality [Willem 1996, Lemma 4.5], we have $u^{*}(x) \rightarrow 0$ as $|x| \rightarrow+\infty$. On the other hand, it follows from $\lim _{s \rightarrow 0^{+}} f(s) / s=0$ that there exists $\Omega \subset \mathbb{R}^{N}$ with $|\Omega|>0$ such that

$$
\left(\frac{f\left(u^{*}(x)\right)}{u^{*}(x)}-l\right)\left|u^{*}(x)\right|^{2}<0
$$

for $x \in \Omega$. If $x \in R^{N} \backslash \Omega$, the left-hand side of (3-16) is nonnegative, by ( $\left.H_{3}\right)$. Thus, we have

$$
\int_{\mathbb{R}^{N}}\left(\frac{f\left(u^{*}\right)}{u^{*}}-l\right)\left|u^{*}\right|^{2} d x<0
$$


This, together with (3-15), yields that there exists $t_{0}=t_{0}\left(u^{*}\right)>0$ such that $I^{\xi}\left(t_{0} u^{*}\right)<0$. Define $\gamma(t)=t t_{0} u^{*}$; then $\gamma(t) \in \Gamma$. By the definition of $c_{1}$, we see that

$$
I^{\xi}(\omega)=c_{1} \leq \max _{0 \leq t \leq 1} I^{\xi}\left(t t_{0} u^{*}\right) \leq \max _{0 \leq t \leq 1} I^{\xi}\left(t t_{0} u\right) \leq \max _{t \geq 0} I^{\xi}(t u)=I^{\xi}(u) .
$$

Since $u$ is arbitrary, we have $I^{\xi}(\omega) \leq G(\xi)$ and (3-13) is shown.

Remark 3.1. By the Gidas-Ni-Nirenberg result [Fei and Yin 2010, Theorem 2 and following remark], 0 is the unique maximum point of $\omega(x)$ in $\mathbb{R}^{N}$. This motivates us to establish a similar result in Lemma 4.5 in Section 4 below.

Finally, we show that the ground energy function $G(\xi)$ is continuous for $\xi \in \bar{\Lambda}$. Here we point out that the continuity of $G(\xi)$ corresponding to the superlinear case of $f(u)$ in (1-3) has been proved in [Wang and Zeng 1997].

Lemma 3.4. $G(\xi)$ is continuous with respect to $\xi \in \bar{\Lambda}$.

Proof. Consider a sequence $\left\{\xi_{j}\right\} \subseteq \bar{\Lambda}$ such that $\xi_{j} \rightarrow \xi_{0} \in \bar{\Lambda}$ as $j \rightarrow+\infty$. Then $V\left(\xi_{j}\right) \rightarrow V\left(\xi_{0}\right), K\left(\xi_{j}\right) \rightarrow K\left(\xi_{0}\right)$ as $j \rightarrow \infty$. Set

$$
\begin{aligned}
& I_{j}(u)=\frac{1}{2} \int_{\mathbb{R}^{N}}|\nabla u|^{2} d x+\frac{V\left(\xi_{j}\right)}{2} \int_{\mathbb{R}^{N}}|u|^{2} d x-K\left(\xi_{j}\right) \int_{\mathbb{R}^{N}} F(u) d x, \\
& I_{0}(u)=\frac{1}{2} \int_{\mathbb{R}^{N}}|\nabla u|^{2} d x+\frac{V\left(\xi_{0}\right)}{2} \int_{\mathbb{R}^{N}}|u|^{2} d x-K\left(\xi_{0}\right) \int_{\mathbb{R}^{N}} F(u) d x,
\end{aligned}
$$

and

$$
\begin{aligned}
& \Gamma_{j}=\left\{\gamma \in C([0,1], E): \gamma(0)=0, I_{j}(\gamma(1))<0\right\}, \\
& \Gamma_{0}=\left\{\gamma \in C([0,1], E): \gamma(0)=0, I_{0}(\gamma(1))<0\right\} .
\end{aligned}
$$

From (3-7) and (3-13), we have

$$
G\left(\xi_{j}\right)=\inf _{\gamma \in \Gamma_{j}} \max _{0 \leq t \leq 1} I_{j}(\gamma(t)) \quad \text { and } \quad G\left(\xi_{0}\right)=\inf _{\gamma \in \Gamma_{0}} \max _{0 \leq t \leq 1} I_{0}(\gamma(t)) .
$$

The proof of the continuity of $G(\xi)$ now proceeds in two steps.

Step 1: $\limsup _{j \rightarrow \infty} G\left(\xi_{j}\right) \leq G\left(\xi_{0}\right)$.

For any fixed path $\gamma(t)$ satisfying $\gamma(0)=0$ and $I_{0}(\gamma(1))<0$, we have $I_{j}(\gamma(1))<$ 0 for large $j$ and

$$
\limsup _{j \rightarrow \infty} G\left(\xi_{j}\right) \leq \limsup _{j \rightarrow \infty} \max _{0 \leq t \leq 1} I_{j}(\gamma(t))=\max _{0 \leq t \leq 1} I_{0}(\gamma(t)) .
$$

Since the path $\gamma(t)$ is arbitrary, this yields

$$
\limsup _{j \rightarrow \infty} G\left(\xi_{j}\right) \leq G\left(\xi_{0}\right) .
$$


Step 2: $\liminf _{j \rightarrow \infty} G\left(\xi_{j}\right) \geq G\left(\xi_{0}\right)$.

We split this step into four parts.

Let $\omega_{j}(x) \in H_{r}^{1}\left(\mathbb{R}^{N}\right)$ satisfy $G\left(\xi_{j}\right)=I_{j}\left(\omega_{j}(x)\right)$ (the existence of $\omega_{j}(x)$ has been shown in Lemma 3.3).

Part 1. $\int_{\mathbb{R}^{N}}\left|\nabla \omega_{j}\right|^{2} d x$ is uniformly bounded with respect to $j$.

According to Pohozaev identity [Willem 1996, Appendix], we have

$$
\frac{N-2}{2 N} \int_{\mathbb{R}^{N}}\left|\nabla \omega_{j}\right|^{2} d x=-\frac{V\left(\xi_{j}\right)}{2} \int_{\mathbb{R}^{N}}\left|\omega_{j}\right|^{2} d x+K\left(\xi_{j}\right) \int_{\mathbb{R}^{N}} F\left(\omega_{j}\right) d x .
$$

This implies

$$
G\left(\xi_{j}\right)=I_{j}\left(\omega_{j}\right)=\frac{1}{N} \int_{\mathbb{R}^{N}}\left|\nabla \omega_{j}\right|^{2} d x .
$$

It follows from (3-17) and (3-18) that there is a positive constant $C$ such that

$$
\int_{\mathbb{R}^{N}}\left|\nabla \omega_{j}\right|^{2} d x \leq C \text { for any } j .
$$

Part 2. $\int_{\mathbb{R}^{N}} \omega_{j}^{2} d x$ has a uniform upper bound independent of $j$.

Note that up to a subsequence, there exists a radial symmetric function $\omega(x)$ such that, as $j \rightarrow \infty$,

$$
\begin{aligned}
& \omega_{j} \rightarrow \omega, \quad \text { weakly in } \mathscr{D}^{1,2}\left(\mathbb{R}^{N}\right), \\
& \omega_{j} \rightarrow \omega, \quad \text { strongly in } L_{\text {loc }}^{t}\left(\mathbb{R}^{N}\right), \quad 1 \leq t<\frac{2 N}{N-2}, \\
& \omega_{j} \rightarrow \omega, \quad \text { almost everywhere in } \mathbb{R}^{N} .
\end{aligned}
$$

By the Strauss inequality [Berestycki and Lions 1983, Lemma A.III, p. 340] for the radial function in $\mathscr{D}^{1,2}\left(\mathbb{R}^{N}\right)$, we have

$$
\left|\omega_{j}(x)\right|^{2} \leq C(N)|x|^{2-N} \int_{\mathbb{R}^{N}}\left|\nabla \omega_{j}(x)\right|^{2} d x, \quad \text { for all }|x| \geq 1,
$$

where the positive constant $C(N)$ only depends on $N$.

Since $f(s) / s \rightarrow 0$ as $s \rightarrow 0$ by the assumption $\left(H_{2}\right)$, we get from (3-23) and the fact that $N \geq 5$ that

$$
\frac{f\left(\omega_{j}(x)\right)}{\omega_{j}(x)} \rightarrow 0 \quad \text { as }|x| \rightarrow \infty \text { uniformly with respect to } j .
$$

This implies that there exists a large number $R>0$ such that

$$
\int_{|x| \geq R}\left(V\left(\xi_{j}\right)-K\left(\xi_{j}\right) \frac{f\left(\omega_{j}\right)}{\omega_{j}}\right)\left|\omega_{j}\right|^{2} d x \geq C \int_{|x| \geq R}\left|\omega_{j}\right|^{2} d x,
$$

where $C>0$ is independent of $R$ and $j$.

It follows from (3-24) and the partial differential equation satisfied by $\omega_{j}$ that 
for large $R$,

$$
\begin{aligned}
C \int_{|x| \geq R}\left|\omega_{j}\right|^{2} d x & \leq \int_{|x| \geq R}\left(V\left(\xi_{j}\right)-K\left(\xi_{j}\right) \frac{f\left(\omega_{j}\right)}{\omega_{j}}\right)\left|\omega_{j}\right|^{2} d x \\
& \leq C \int_{|x| \leq R}\left|\omega_{j}\right|^{2} d x \rightarrow C \int_{|x| \leq R}|\omega|^{2} d x \quad \text { as } j \rightarrow \infty
\end{aligned}
$$

Combining (3-24) with (3-25) yields that $\int_{\mathbb{R}^{N}}\left|\omega_{j}\right|^{2} d x$ has a uniform supper bound with respect to $j$. Thus $\omega \in L^{2}\left(\mathbb{R}^{N}\right)$ and further $\omega \in H^{1}\left(\mathbb{R}^{N}\right)$. Moreover, $\omega$ is a solution of the equation

$$
-\triangle \omega(x)+V\left(\xi_{0}\right) \omega(x)=K\left(\xi_{0}\right) f(\omega), \quad x \in \mathbb{R}^{N} .
$$

Part 3. $\int_{\mathbb{R}^{N}}\left|\omega_{j}\right|^{2} d x$ has a uniform positive lower bound with respect to $j$.

We now show that $\int_{\mathbb{R}^{N}}\left|\omega_{j}\right|^{2} d x$ has a uniform positive lower bound with respect to $j$. If so, this assertion together with (3-21) and (3-25) will yield

$$
\omega \not \equiv 0
$$

Note that $V\left(\xi_{0}\right) / K\left(\xi_{0}\right)<l$ and $V\left(\xi_{j}\right) \rightarrow V\left(\xi_{0}\right), K\left(\xi_{j}\right) \rightarrow K\left(\xi_{0}\right)$ as $j \rightarrow \infty$. Thus we can choose a fixed small number $\eta>0$ satisfying

$$
\frac{V\left(\xi_{0}\right)-\eta}{K\left(\xi_{0}\right)+\eta}<l,
$$

and, for large $j$,

$$
V\left(\xi_{j}\right)>V\left(\xi_{0}\right)-\eta, \quad K\left(\xi_{j}\right)<K\left(\xi_{0}\right)+\eta .
$$

Let $m_{0}$ be the ground energy of the functional

$$
H^{1}\left(\mathbb{R}^{N}\right) \ni u \mapsto \frac{1}{2} \int_{\mathbb{R}^{N}}|\nabla u|^{2} d x+\frac{V\left(\xi_{0}\right)-\eta}{2} \int_{\mathbb{R}^{N}}|u|^{2} d x-\left(K\left(\xi_{0}\right)+\eta\right) \int_{\mathbb{R}^{N}} F(u) d x
$$

in the Nehari manifold $\mathcal{M}^{\eta}$, which is defined as

$$
\begin{aligned}
\mathcal{M}^{\eta}=\left\{u \in H^{1}\left(\mathbb{R}^{N}\right) \backslash\{0\}:\right. & \\
& \left.\int_{\mathbb{R}^{N}}|\nabla u|^{2} d x+\left(V\left(\xi_{0}\right)-\eta\right) \int_{\mathbb{R}^{N}}|u|^{2} d x=\left(K\left(\xi_{0}\right)+\eta\right) \int_{\mathbb{R}^{N}} f(u) u d x\right\} .
\end{aligned}
$$

By (3-28) and the similar proof on Lemma 3.3, one can show that $m_{0}$ is achieved and is positive (in the arguments of Lemma 3.3, we have used the condition $V(\xi) / K(\xi)<l$ parallel to $(3-28))$. 
Consider the function

$g_{j}(t)$

$=\int_{\mathbb{R}^{N}}\left|\nabla\left(t \omega_{j}\right)\right|^{2} d x+\left(V\left(\xi_{0}\right)-\eta\right) \int_{\mathbb{R}^{N}}\left|t \omega_{j}\right|^{2} d x-\left(K\left(\xi_{0}\right)+\eta\right) \int_{\mathbb{R}^{N}} f\left(t \omega_{j}\right) t \omega_{j} d x$.

Recalling that $\lim _{s \rightarrow 0} F(s) / s^{2}=\lim _{s \rightarrow 0} f(s) /(2 s)=0$, we get $g_{j}(t)>0$ for $0<t \ll 1$. In addition, by (3-29) we get $g_{j}(1)<I_{j}^{\prime}\left(\omega_{j}\right) \omega_{j}=0$. Therefore there exists a $t_{j} \in(0,1)$ such that $g_{j}\left(t_{j} \omega_{j}\right)=0$, that is,

$$
\begin{aligned}
& \frac{1}{2} \int_{\mathbb{R}^{N}}\left|\nabla\left(t_{j} \omega_{j}\right)\right|^{2} d x \\
& \quad+\frac{V\left(\xi_{0}\right)-\eta}{2} \int_{\mathbb{R}^{N}}\left|t_{j} \omega_{j}\right|^{2} d x-\left(K\left(\xi_{0}\right)+\eta\right) \int_{\mathbb{R}^{N}} F\left(t_{j} \omega_{j}\right) d x \geq m_{0} .
\end{aligned}
$$

Set

$$
h_{j}(t)=\frac{1}{2} \int_{\mathbb{R}^{N}}\left|\nabla\left(t \omega_{j}\right)\right|^{2} d x+\frac{V\left(\xi_{j}\right)}{2} \int_{\mathbb{R}^{N}}\left|t \omega_{j}\right|^{2} d x-K\left(\xi_{j}\right) \int_{\mathbb{R}^{N}} F\left(t \omega_{j}\right) d x .
$$

It follows from a direct computation and the assumption $\left(H_{3}\right)$ that, for $t \in(0,1]$,

$$
\begin{aligned}
h_{j}^{\prime}(t) & =t \int_{\mathbb{R}^{N}}\left|\nabla \omega_{j}\right|^{2} d x+t V\left(\xi_{j}\right) \int_{\mathbb{R}^{N}}\left|\omega_{j}\right|^{2} d x-K\left(\xi_{j}\right) \int_{\mathbb{R}^{N}} f\left(t \omega_{j}\right) \omega_{j} d x \\
& \geq 0
\end{aligned}
$$

Combining (3-29), (3-30), and (3-31), we obtain, for large $j$,

$$
I_{j}\left(\omega_{j}\right) \geq m_{0} .
$$

Together with (3-18), this yields, for large $j$,

$$
\frac{1}{N} \int_{\mathbb{R}^{N}}\left|\nabla \omega_{j}\right|^{2} d x=I_{j}\left(\omega_{j}\right) \geq m_{0}
$$

In addition, since

$$
\left(\frac{F(s)}{s^{2}}\right)^{\prime}=\frac{f(s) s-2 F(s)}{s^{3}} \geq 0 \text { and } \lim _{s \rightarrow+\infty} \frac{F(s)}{s^{2}}=\lim _{s \rightarrow+\infty} \frac{f(s)}{2 s}=\frac{l}{2},
$$

we have

$$
0 \leq \frac{F(s)}{s^{2}} \leq \frac{l}{2}, \quad s \neq 0 .
$$

Therefore, by (3-32), (3-33), and the Pohozaev identity we find that

$$
0<C \leq \frac{N-2}{2 N} \int_{\mathbb{R}^{N}}\left|\nabla \omega_{j}\right|^{2} d x \leq C \int_{\mathbb{R}^{N}} \omega_{j}^{2} d x,
$$


where $C$ is a generic positive constant independent of $j$, that is, $\int_{\mathbb{R}^{N}}\left|\omega_{j}\right|^{2} d x$ have a uniform positive lower bound with respect to $j$.

Part 4. $\lim _{j \rightarrow \infty} \int_{\mathbb{R}^{N}} F\left(\omega_{j}\right) d x=\int_{\mathbb{R}^{N}} F(\omega) d x$.

In order to show

$$
\lim _{j \rightarrow \infty} \int_{\mathbb{R}^{N}} F\left(\omega_{j}\right) d x=\int_{\mathbb{R}^{N}} F(\omega) d x,
$$

then by (3-21) we only need to prove:

For any given $\delta>0$, there exists $R>0$ such that, for large $j$,

$$
\left|\int_{\mathbb{R}^{N} \backslash B_{R}} F\left(\omega_{j}\right) d x\right|<\delta .
$$

In fact, if we set $\eta_{R}$ to be a smooth cut-off function such that $\eta_{R}=0$ for $|x| \leq \frac{R}{2}$, $\eta_{R}=1$ for $|x| \geq R$ and $|\nabla \eta| \leq \frac{4}{R}$, then multiplying by $\eta_{R} \omega_{j}$ the equation

$$
-\triangle \omega_{j}+V\left(\xi_{j}\right) \omega_{j}=K\left(\xi_{j}\right) f\left(\omega_{j}\right), \quad x \in \mathbb{R}^{N},
$$

yields, for large $R$ and $j$,

$$
C \int_{|x| \geq R}\left(\left|\nabla \omega_{j}\right|^{2}+\left|\omega_{j}\right|^{2}\right) d x \leq \frac{C}{R} \rightarrow 0 \quad \text { as } R \rightarrow+\infty,
$$

which means that (3-36) and further (3-35) hold.

Finally, we show $\liminf _{j \rightarrow \infty} G\left(\xi_{j}\right) \geq G\left(\xi_{0}\right)$. In view of (3-35), (3-26)-(3-27) and the fact that $G\left(\xi_{0}\right)$ is the ground energy of the functional $I_{0}$, we have (3-37) $\liminf _{j \rightarrow \infty} G\left(\xi_{j}\right)$

$$
=\liminf _{j \rightarrow \infty}\left\{\frac{1}{2} \int_{\mathbb{R}^{N}}\left(\left|\nabla \omega_{j}\right|^{2}+V\left(\xi_{j}\right)\left|\omega_{j}\right|^{2}\right) d x-K\left(\xi_{j}\right) \int_{\mathbb{R}^{N}} F\left(\omega_{j}\right) d x\right\} \geq G\left(\xi_{0}\right) .
$$

Thus the continuity of $G(\xi)$ is derived from (3-17) and (3-37), that is, Lemma 3.4 is proved.

\section{The proof of Theorem 1.1}

At first, we intend to obtain an upper bound estimate of the critical value $c_{\varepsilon}$ corresponding to the functional $I_{\varepsilon}(u)$ defined in Section 2, which will play a crucial role in establishing the concentration and decay estimates of solution $u_{\varepsilon}$ to Equation (2-1). From the decay estimates of $u_{\varepsilon}$ we can show $g_{\varepsilon}\left(x, u_{\varepsilon}\right) \equiv K(x) f\left(u_{\varepsilon}\right)$ in $\mathbb{R}^{N} \backslash \Lambda$ and subsequently complete the proof of Theorem 1.1.

Lemma 4.1. Under the hypotheses $\left(H_{1}\right)-\left(H_{4}\right)$, and with $c_{0}$ as in $\left(H_{4}\right)$, we have, for small $\varepsilon>0$,

$$
c_{\varepsilon} \leq\left(c_{0}+o_{\varepsilon}(1)\right) \varepsilon^{N}
$$


Proof. For $\xi \in \Lambda$, choose $R>0$ such that $B_{R}(\xi) \subset \Lambda$. Define a smooth cut-off function $\eta: \mathbb{R}^{+} \rightarrow \mathbb{R}^{+}$satisfying $\eta(t)=1$ if $0 \leq t \leq \frac{R}{4}, \eta(t)=0$ if $t \geq \frac{R}{2}$ and $\left|\eta^{\prime}(t)\right| \leq \frac{8}{R}$. Set

$$
w_{\varepsilon}(x)=\eta(|x-\xi|) \omega\left(\frac{x-\xi}{\varepsilon}\right),
$$

where $\omega(x)=\omega(V(\xi), K(\xi) ; x)$ is the solution of $(1-3)$.

Noting that $w_{\varepsilon}$ is compactly supported in $\Lambda$, one can get $G_{\varepsilon}\left(x, t w_{\varepsilon}\right)=0$ for all $t \geq 0$ and $x \in \Lambda$, where $G_{\varepsilon}(x, u)$ is the function defined in (2-2). Then as in the argument in (2-11), there exists a sufficiently large $T>0$ such that $I_{\varepsilon}\left(T w_{\varepsilon}\right)<$ 0 . This implies that the path $\gamma_{\varepsilon}(t)=\left\{t T w_{\varepsilon}: t \in[0,1]\right\}$ is an element of $\Gamma_{\varepsilon}$ satisfying $c_{\varepsilon} \leq \max _{0 \leq t \leq 1} I_{\varepsilon}\left(\gamma_{\varepsilon}(t)\right)$. Also, similar to the proof of (2-10), we infer that $I_{\varepsilon}\left(t T w_{\varepsilon}\right)=\varepsilon^{N}\left(I^{\xi}(t T w)+o_{\varepsilon}(1)\right)$. Hence

$\max _{0 \leq t \leq 1} I_{\varepsilon}\left(\gamma_{\varepsilon}(t)\right)=\max _{0 \leq t \leq 1} I_{\varepsilon}\left(t T w_{\varepsilon}\right)=\varepsilon^{N}\left(\max _{0 \leq t \leq 1} I^{\xi}(t T w)+o_{\varepsilon}(1)\right)=\varepsilon^{N}\left(G(\xi)+o_{\varepsilon}(1)\right)$.

Since $\xi$ is arbitrary and the smallness of $\varepsilon$ is independent of the choice of $\xi$, then Lemma 4.1 is proved.

The next result illustrates that the maximum of $u_{\varepsilon}$ on $\bar{\Lambda}$ has a uniform positive lower bound.

Lemma 4.2. Let $x_{\varepsilon}$ be the maximum point of $u_{\varepsilon}$ on $\bar{\Lambda}$, then there exists a positive constant $C$ independent of $\varepsilon$ such that

$$
u_{\varepsilon}\left(x_{\varepsilon}\right) \geq C .
$$

Proof. By $\left(H_{2}\right)$ and $\left(H_{3}\right)$, for any $\delta>0$, there exists $C_{\delta}>0$ such that $f(s) \leq$ $\delta s+C_{\delta}|s|^{2}$. From $I_{\varepsilon}^{\prime}\left(u_{\varepsilon}\right) u_{\varepsilon}=0$, one has, for small $\delta$ and $\varepsilon$,

$$
\begin{aligned}
\left\|u_{\varepsilon}\right\|_{\varepsilon}^{2} & =\int_{\Lambda} K(x) f\left(x, u_{\varepsilon}\right) u_{\varepsilon} d x+\int_{\mathbb{R}^{N} \backslash \Lambda} g_{\varepsilon}\left(x, u_{\varepsilon}\right) u_{\varepsilon} d x \\
& \leq \frac{1}{2}\left\|u_{\varepsilon}\right\|_{\varepsilon}^{2}+C\left\|u_{\varepsilon}\right\|_{\varepsilon}^{2} \max _{\bar{\Lambda}} u_{\varepsilon} .
\end{aligned}
$$

Obviously this means that there exists a positive number $C$ independent of $\varepsilon$ such that $u_{\varepsilon}\left(x_{\varepsilon}\right) \geq C$ holds true due to $\left\|u_{\varepsilon}\right\|_{\varepsilon} \neq 0$, then the proof of Lemma 4.2 is completed.

Note that since $f(s)$ is asymptotically linear, then in the general case, there is no number $\theta>0$ such that $(2+\theta) F(s) \leq f(s) s$ for any $s>0$, here $F(s)=\int_{0}^{s} f(\tau) d \tau$. However, in the superlinear case, this property of $(2+\theta) F(s) \leq f(s) s$ with $\theta>0$ play a crucial role in obtaining the uniform boundedness of $\varepsilon^{-N}\left\|u_{\varepsilon}\right\|_{\varepsilon}$ from (4-1), which will be used to derive the decay estimate of $u_{\varepsilon}$ at infinity and the concentration of $u_{\varepsilon}$ as $\varepsilon \rightarrow 0$ (one can see the details in [Fei and Yin 2010] and some references therein). To overcome this kind of difficulty, next we will use some different 
ingredients (motivated by the proofs of Lemmas 2.2-2.3) to treat the uniform boundedness of $\varepsilon^{-N}\left\|u_{\varepsilon}\right\|_{\varepsilon}$.

Lemma 4.3. There exists a positive constant $C$ independent of small $\varepsilon$ such that

$$
\varepsilon^{-N} \int_{\mathbb{R}^{N}}\left(\varepsilon^{2}\left|\nabla u_{\varepsilon}\right|^{2}+V(x)\left|u_{\varepsilon}\right|^{2}\right) d x \leq C
$$

namely,

$$
\int_{\mathbb{R}^{N}}\left(\left|\nabla v_{\varepsilon}\right|^{2}+V\left(\varepsilon x+x_{\varepsilon}\right)\left|v_{\varepsilon}\right|^{2}\right) d x \leq C,
$$

where $v_{\varepsilon}(x)=u_{\varepsilon}\left(\varepsilon x+x_{\varepsilon}\right)$ and the meaning of $x_{\varepsilon}$ is given in Lemma 4.2.

Proof. For convenience we will use the notation $\left\|v_{\varepsilon}\right\|$ with

$$
\left\|v_{\varepsilon}\right\|=\left(\int_{\mathbb{R}^{N}}\left(\left|\nabla v_{\varepsilon}\right|^{2}+V\left(\varepsilon x+x_{\varepsilon}\right)\left|v_{\varepsilon}\right|^{2}\right) d x\right)^{1 / 2} .
$$

If (4-4) does not hold, there exists a sequence of functions $v_{n}(x) \equiv u_{\varepsilon_{n}}\left(\varepsilon_{n} x+x_{n}\right)$ such that $\left\|v_{n}\right\| \rightarrow+\infty$ as $n \rightarrow \infty$ and $v_{n}(x)$ satisfies

$$
\begin{aligned}
& -\Delta v_{n}+V\left(\varepsilon_{n} x+x_{n}\right) v_{n} \\
& \quad=\chi_{\Omega_{n}}(x) K\left(\varepsilon_{n} x+x_{n}\right) f\left(v_{n}\right)+\left(1-\chi_{\Omega_{n}}(x)\right) g_{\varepsilon_{n}}\left(\varepsilon_{n} x+x_{n}, v_{n}\right),
\end{aligned}
$$

where $\Omega_{n} \equiv \varepsilon_{n}^{-1}\left(\Lambda-x_{n}\right)$ and $x_{n} \equiv x_{\varepsilon_{n}} \in \bar{\Lambda}$.

Set $\omega_{n}=v_{n} /\left\|v_{n}\right\|$, then $\left\|\omega_{n}\right\|=1$ and $\omega_{n}(x)$ satisfies

$$
\begin{aligned}
-\Delta \omega_{n} & +V\left(\varepsilon_{n} x+x_{n}\right) \omega_{n} \\
& =\chi_{\Omega_{n}}(x) K\left(\varepsilon_{n} x+x_{n}\right) \frac{f\left(v_{n}\right)}{v_{n}} \omega_{n}+\left(1-\chi_{\Omega_{n}}(x)\right) \frac{g_{\varepsilon_{n}}\left(\varepsilon_{n} x+x_{n}, v_{n}\right)}{\left\|v_{n}\right\|} .
\end{aligned}
$$

We rewrite (4-6) as

$$
-\Delta \omega_{n}=a_{n}(x) \omega_{n}
$$

where

$a_{n}(x)=-V\left(\varepsilon_{n} x+x_{n}\right)+\chi_{\Omega_{n}}(x) K\left(\varepsilon_{n} x+x_{n}\right) \frac{f\left(v_{n}\right)}{v_{n}}+\left(1-\chi_{\Omega_{n}}(x)\right) \frac{g_{\varepsilon_{n}}\left(\varepsilon_{n} x+x_{n}, v_{n}\right)}{v_{n}}$.

For any fixed and bounded smooth domain $\Omega \subset \mathbb{R}^{N}$ and fixed $\alpha \in(0,1)$, due to $\left\|a_{n}(x)\right\|_{L^{\infty}(\Omega)} \leq C(\Omega)$, it follows from $\left\|\omega_{n}\right\|=1$ and the elliptic equation (4-7) that $\left\|\omega_{n}\right\|_{C^{1, \alpha}(\bar{\Omega})} \leq C(\Omega, \alpha)$, where the positive constants $C(\Omega)$ and $C(\Omega, \alpha)$ depend on $\Omega$ and $\Omega, \alpha$ respectively. Therefore, for fixed $\beta \in(0, \alpha)$, there exists a subsequence still denoted by $\left\{\omega_{n}\right\}$ and a function $\omega$ such that $\omega_{n} \rightarrow \omega$ in $C^{1, \beta}(\bar{\Omega})$.

In particular, for a series of closed ball sequences $B_{k}(0), k=1,2, \ldots$, then there exists a subsequence $\left\{\omega_{1 n}\right\}$ and a function $\omega_{1}$ such that $\omega_{1 n} \rightarrow \omega_{1}$ in $C^{1, \beta}\left(B_{1}(0)\right)$, and there exists a subsequence $\left\{\omega_{(k+1) n}\right\} \subseteq\left\{\omega_{k n}\right\}$ and a function $\omega_{k+1}$ such that 
$\omega_{(k+1) n} \rightarrow \omega_{k+1}$ in $C^{1, \beta}\left(B_{k+1}(0)\right)$ as $n \rightarrow \infty$ for $k \geq 1$. By the diagonal process, one knows that there exists a subsequence still denoted by $\left\{\omega_{n}\right\}$ and a function $\omega$ such that $\omega_{n} \rightarrow \omega$ in $C_{\text {loc }}^{1, \beta}\left(\mathbb{R}^{N}\right)$ as $n \rightarrow+\infty$. Of course, $\lim _{n \rightarrow \infty} \omega_{n}(x)=\omega(x)$ holds for $x \in \mathbb{R}^{N}$.

Let $x_{n} \rightarrow x_{0} \in \bar{\Lambda}$. We consider two cases.

Case I: $\lim _{n \rightarrow \infty} \operatorname{dist}\left(x_{n}, \partial \Lambda\right) / \varepsilon_{n}=+\infty$.

In this case, by taking a subsequence, we can assume $x_{n} \in \Lambda$. Hence $0 \in$ $\Omega_{n}$ and $\lim _{n \rightarrow \infty} \operatorname{dist}\left(0, \partial \Omega_{n}\right)=\lim _{n \rightarrow \infty} \operatorname{dist}\left(x_{n}, \partial \Lambda\right) / \varepsilon_{n}=+\infty$, which leads to $\lim _{n \rightarrow \infty} \Omega_{n}=\mathbb{R}^{N}$.

For any fixed $\varphi \in C_{0}^{\infty}\left(\mathbb{R}^{N}\right)$, there holds supp $\varphi \subseteq \Omega_{n}$ for lager $n$. Multiplying $\varphi$ on two hand sides of (4-6) and integrating by parts yield, for large $n$,

$$
\int\left[\nabla \omega_{n} \nabla \varphi+V\left(\varepsilon_{n} x+x_{n}\right) \omega_{n} \varphi\right] d x=\int K\left(\varepsilon_{n} x+x_{n}\right) \frac{f\left(v_{n}\right)}{v_{n}} \omega_{n} \varphi d x .
$$

Note that

$$
\lim _{n \rightarrow \infty} \int\left[\nabla \omega_{n} \nabla \varphi+V\left(\varepsilon_{n} x+x_{n}\right) \omega_{n} \varphi\right] d x=\int\left[\nabla \omega \nabla \varphi+V\left(x_{0}\right) \omega \varphi\right] d x .
$$

Next we show that

$$
\lim _{n \rightarrow \infty} \int K\left(\varepsilon_{n} x+x_{n}\right) \frac{f\left(v_{n}\right)}{v_{n}} \omega_{n} \varphi d x=\int K\left(x_{0}\right) l \omega \varphi d x .
$$

Define the set $A=\left\{x \in \mathbb{R}^{N}: \lim _{n \rightarrow \infty} v_{n}(x)=+\infty\right\}$ and let $A^{c}=\mathbb{R}^{N} \backslash A$. If $x \in A$, then $\lim _{n \rightarrow \infty} f\left(v_{n}(x)\right) / v_{n}(x)=l$. If $x \in A^{c}$, since $\lim _{n \rightarrow \infty}\left\|v_{n}\right\|=+\infty$, we have $\omega(x)=\lim _{n \rightarrow \infty} \omega_{n}(x)=\liminf _{n \rightarrow \infty} v_{n}(x) /\left\|v_{n}\right\|=0$.

On the other hand, since $K\left(\varepsilon_{n} x+x_{n}\right)$ is uniformly bounded for $x \in \operatorname{supp} \varphi$ with respect to $n$ and $f(s) / s$ is also bounded, we have

(4-11) $\lim _{n \rightarrow \infty} \int K\left(\varepsilon_{n} x+x_{n}\right) \frac{f\left(v_{n}\right)}{v_{n}} \omega_{n} \varphi d x=\lim _{n \rightarrow \infty} \int K\left(\varepsilon_{n} x+x_{n}\right) \frac{f\left(v_{n}\right)}{v_{n}} \omega \varphi d x$.

Therefore,

$$
\lim _{n \rightarrow \infty} \int_{\operatorname{supp} \varphi \cap A} K\left(\varepsilon_{n} x+x_{n}\right) \frac{f\left(v_{n}\right)}{v_{n}} \omega \varphi d x=\int_{\operatorname{supp} \varphi \cap A} K\left(x_{0}\right) l \omega \varphi d x .
$$

In addition, obviously,

$$
\text { (4-13) } \lim _{n \rightarrow \infty} \int_{\operatorname{supp} \varphi \cap A^{c}} K\left(\varepsilon_{n} x+x_{n}\right) \frac{f\left(v_{n}\right)}{v_{n}} \omega \varphi d x=0=\int_{\operatorname{supp} \varphi \cap A^{c}} K\left(x_{0}\right) l \omega \varphi d x \text {. }
$$

Collecting (4-11)-(4-13) yields (4-10).

From (4-8)-(4-10), we arrive at

$$
\int_{\mathbb{R}^{N}} \nabla \omega \nabla \varphi+V\left(x_{0}\right) \omega \varphi=\int_{\mathbb{R}^{N}} K\left(x_{0}\right) l \omega \varphi,
$$


which means that $\omega$ solves

$$
-\Delta \omega+V\left(x_{0}\right) \omega=K\left(x_{0}\right) l \omega .
$$

Case II: $\liminf _{n \rightarrow \infty} \operatorname{dist}\left(x_{n}, \partial \Lambda\right) / \varepsilon_{n} \leq C$.

In this case, we can show that $x_{0} \in \partial \Lambda$. Thus, up to a rotation, we can obtain $\lim _{n \rightarrow \infty} \Omega_{n}=\left\{x \in \mathbb{R}^{N}: x_{1}<0\right\}$. Similarly to Case I, we conclude that the function $\omega(x)$ satisfies

$$
-\Delta \omega+V\left(x_{0}\right) \omega=K\left(x_{0}\right) l \omega \chi_{\left\{x_{1}<0\right\}}(x) .
$$

In Case I or Case II, for any fixed bounded domain $M \subset \mathbb{R}^{N}$ or $M \subset\left\{x \in \mathbb{R}^{N}\right.$ : $\left.x_{1}<0\right\}$ we have

$$
\begin{aligned}
\int_{M}\left[|\nabla \omega|^{2}+V\left(x_{0}\right) \omega^{2}\right] d x & =\lim _{n \rightarrow \infty} \int_{M}\left[\left|\nabla \omega_{n}\right|^{2}+V\left(\varepsilon_{n} x+x_{n}\right) \omega_{n}^{2}\right] d x \\
& \leq \int_{\mathbb{R}^{N}}\left[\left|\nabla \omega_{n}\right|^{2}+V\left(\varepsilon_{n} x+x_{n}\right) \omega_{n}^{2}\right] d x=1 ;
\end{aligned}
$$

then

$$
\int_{\mathbb{R}^{N}}\left[|\nabla \omega|^{2}+V\left(x_{0}\right) \omega^{2}\right] d x \leq 1,
$$

which means $\omega \in H^{1}\left(\mathbb{R}^{N}\right)$ due to $V\left(x_{0}\right)>0$.

It follows the equations (4-15)-(4-16), together with (4-17), the fact that $\omega \geq$ 0 , regularity theory and the strong maximum principle for second-order elliptic equations, that we can get $\omega(x) \in C^{2, \gamma}\left(\mathbb{R}^{N}\right)$ in Case I and $\omega(x) \in C^{1, \alpha}\left(\mathbb{R}^{N}\right)$ for any $\alpha \in(0,1)$ in Case II, and $\omega(x)>0$ with $\omega(x) \rightarrow 0$ as $|x| \rightarrow \infty$. However, this is contradictory with the conclusion of Lemma 2.3. Thus (4-15) and (4-16) have no nontrivial nonnegative solutions. Lemma 4.3 is proved.

Next we assert that the maximum point of $u_{\varepsilon}$ on $\bar{\Lambda}$ must lie in the interior of $\Lambda$.

Lemma 4.4. $\lim _{\varepsilon \rightarrow 0} \max _{\partial \Lambda} u_{\varepsilon}=0$.

Proof. To prove this, we argue by contradiction assuming that there exists a sequence $\varepsilon_{n} \rightarrow 0$ as $n \rightarrow \infty$ such that for each $n$,

$$
\max _{\partial \Lambda} u_{\varepsilon_{n}} \geq C>0 .
$$

Let $x_{n} \in \partial \Lambda$ such that $u_{\varepsilon_{n}}\left(x_{n}\right)=\max _{\partial \Lambda} u_{\varepsilon_{n}}$ and $x_{n} \rightarrow x_{0} \in \partial \Lambda$ as $n \rightarrow \infty$. Define $v_{n}(x)=u_{\varepsilon_{n}}\left(\varepsilon_{n} x+x_{n}\right)$, then $v_{n}(0) \geq C$ and $v_{n}(x)$ satisfies

$$
\begin{aligned}
-\Delta v_{n}+V & \left(\varepsilon_{n} x+x_{n}\right) v_{n} \\
& =\chi_{\Omega_{n}}(x) K\left(\varepsilon_{n} x+x_{n}\right) f\left(v_{n}\right)+\left(1-\chi_{\Omega_{n}}(x)\right) g_{\varepsilon_{n}}\left(\varepsilon_{n} x+x_{n}, v_{n}\right),
\end{aligned}
$$

where $\Omega_{n} \equiv \varepsilon_{n}^{-1}\left(\Lambda-x_{n}\right)$. 
By (4-4), there holds

$$
\int_{\mathbb{R}^{N}}\left|\nabla v_{n}\right|^{2} d x \leq C
$$

which deduces that for large $n$, for any fixed $R>0$, there exists a positive constant $C(R)$ depending on $R$ such that

$$
\int_{B_{R}(0)}\left(\left|\nabla v_{n}\right|^{2}+v_{n}^{2}\right) d x \leq C(R) .
$$

In terms of this and (4-19), as in the proof of Lemma 4.3, there exists some nonnegative function $v(x)$ such that $v_{n} \rightarrow v(x)$ in $C_{\text {loc }}^{2}\left(\mathbb{R}^{N}\right)$ and $v(x)$ satisfies

$$
-\Delta v+V\left(x_{0}\right) v=K\left(x_{0}\right) \chi_{\left\{x_{1}<0\right\}} f(v), \quad x=\left(x_{1}, x^{\prime}\right) \in \mathbb{R}^{N} .
$$

Note that $v_{n}(0) \geq C$, then $v(0) \geq C$ and further $v(x)>0$ in $\mathbb{R}^{N}$ by the maximum principle and Equation (4-20).

On the other hand, acting the test function $\partial_{x_{1}} v$ on (4-20) yields

$$
\int_{\mathbb{R}^{N-1}} F\left(v\left(0, x^{\prime}\right)\right) d x^{\prime}=0,
$$

which leads to $v\left(0, x^{\prime}\right)=0$. However, this is impossible due to $v(x)>0$ in $\mathbb{R}^{N}$. Thus Lemma 4.4 is proved.

Lemma 4.5. For small $\varepsilon, u_{\varepsilon}$ possesses at most one maximum point $x_{\varepsilon}$ on $\bar{\Lambda}$ and $G\left(x_{\varepsilon}\right) \rightarrow c_{0}$ as $\varepsilon \rightarrow 0$.

Proof. First, we prove $G\left(x_{\varepsilon}\right) \rightarrow c_{0}$ as $\varepsilon \rightarrow 0$.

If not, we have $\lim \sup _{\varepsilon \rightarrow 0} G\left(x_{\varepsilon}\right)>c_{0}$. Let $x_{\varepsilon_{j}} \rightarrow x_{0} \in \bar{\Lambda}$; then $\lim _{j \rightarrow \infty} G\left(x_{\varepsilon_{j}}\right)=$ $\lim \sup _{\varepsilon \rightarrow 0} G\left(x_{\varepsilon}\right)>c_{0}$, which means $G\left(x_{0}\right)>c_{0}$.

Set $v_{j}(x)=u_{\varepsilon_{j}}\left(\varepsilon_{j} x+x_{\varepsilon_{j}}\right)$. Then $v_{j}$ solves

$$
\begin{aligned}
-\Delta v_{j}+ & V\left(\varepsilon_{j} x+x_{\varepsilon_{j}}\right) v_{j} \\
& =\chi_{\Omega_{j}}(x) K\left(\varepsilon_{j} x+x_{\varepsilon_{j}}\right) f\left(v_{j}\right)+\left(1-\chi_{\Omega_{j}}(x)\right) g_{\varepsilon_{j}}\left(\varepsilon_{j} x+x_{\varepsilon_{j}}, v_{j}\right) .
\end{aligned}
$$

As before, we can show that $v_{j}$ converges in $C_{\text {loc }}^{1, \alpha}\left(\mathbb{R}^{N}\right)$ for $\alpha \in(0,1)$ to some function $v_{0}$ that satisfies

$$
-\Delta v_{0}+V\left(x_{0}\right) v_{0}=K\left(x_{0}\right) f\left(v_{0}\right), \quad x \in \mathbb{R}^{N}
$$

or

$$
-\Delta v_{0}+V\left(x_{0}\right) v_{0}=K\left(x_{0}\right) \chi_{\left\{x_{1}<0\right\}} f\left(v_{0}\right), \quad x=\left(x_{1}, x^{\prime}\right) \in \mathbb{R}^{N} .
$$

The case of (4-23) can be excluded by the same argument as in Lemma 4.4, so we focus on the case of (4-22). 
Set

$$
\begin{aligned}
& J_{\varepsilon_{j}}\left(v_{j}\right)=\frac{1}{2} \int_{\mathbb{R}^{N}}\left|\nabla v_{j}\right|^{2} d x+V\left(\varepsilon_{j} x+x_{\varepsilon_{j}}\right)\left|v_{j}\right|^{2} d x \\
& -\int_{\left(\Lambda-x_{\varepsilon_{j}}\right) / \varepsilon_{j}} K\left(\varepsilon_{j} x+x_{\varepsilon_{j}}\right) F\left(v_{j}\right) d x-\int_{\mathbb{R}^{N} \backslash\left(\Lambda-x_{\varepsilon_{j}}\right) / \varepsilon_{j}} G\left(\varepsilon_{j} x+x_{\varepsilon_{j}}, v_{j}\right) d x .
\end{aligned}
$$

By invoking Lemma 2.2 in [del Pino and Felmer 1996] together with $2 F(s) \leq$ $f(s) s$, we conclude that

$$
\liminf _{j \rightarrow \infty} J_{\varepsilon_{j}}\left(v_{j}\right) \geq I^{x_{0}}\left(v_{0}\right) .
$$

This, together with (4-1), yields

$$
c_{0} \geq \liminf _{j \rightarrow \infty} \varepsilon_{j}^{-N} I_{\varepsilon_{j}}\left(u_{\varepsilon_{j}}\right)=\liminf _{j \rightarrow \infty} J_{\varepsilon_{j}}\left(v_{j}\right) \geq I^{x_{0}}\left(v_{0}\right) \geq G\left(x_{0}\right)>c_{0},
$$

which leads to a contradiction.

In addition, using the arguments in [del Pino and Felmer 1996, p. 133], we can show that $u_{\varepsilon}$ possesses at most one maximum point $x_{\varepsilon}$ on $\bar{\Lambda}$. We omit the details. This concludes the proof of Lemma 4.5.

Next we establish a compactness result for $u_{\varepsilon}$ which will be crucial to derive the decay of $u_{\varepsilon}(x)$ as $|x| \rightarrow \infty$.

Lemma 4.6. For any $v>0$, there exist $\rho_{0}(v), \varepsilon_{0}(v)>0$ such that for $\rho>\rho_{0}(v)$, $\varepsilon<\varepsilon_{0}(v)$, then

$$
\operatorname{dist}\left(x_{\varepsilon}, M\right)<v,
$$

and

$$
\varepsilon^{-N} \int_{\mathbb{R}^{N} \backslash B_{\varepsilon \rho}\left(x_{\varepsilon}\right)}\left(\varepsilon^{2}\left|\nabla u_{\varepsilon}\right|^{2}+V(x)\left|u_{\varepsilon}\right|^{2}\right) d x<v,
$$

where $M=\left\{\xi \in \Lambda: G(\xi)=c_{0}\right\}$, and the meaning of $c_{0}$ is given in (1-8).

Proof. Since the first conclusion can be directly derived from Lemma 4.5, then it suffices to prove (4-27).

As a consequence of Lemma 4.5 and the assumption on $G(x)$ in $\left(H_{4}\right)$, we have $d=\inf _{n} \operatorname{dist}\left(x_{n}, \partial \Lambda\right)>0$ and $\Lambda_{n}=\left(\Lambda-x_{n}\right) / \varepsilon_{n} \supset B_{d / \varepsilon_{n}} \equiv B_{\tilde{\rho}_{n}}$.

If (4-27) does not hold, then we can assume that there exist $\nu_{0}>0, \tilde{\rho}_{n}>\rho_{n} \rightarrow$ $+\infty, \varepsilon_{n} \rightarrow 0$ as $n \rightarrow \infty$ such that

$$
\mathscr{T} \equiv \varepsilon_{n}^{-N} \int_{\mathbb{R}^{N} \backslash B_{\varepsilon_{n} \rho_{n}}\left(x_{n}\right)}\left(\varepsilon^{2}\left|\nabla u_{n}\right|^{2}+V(x)\left|u_{n}\right|^{2}\right) d x>v_{0},
$$

where $x_{n} \equiv x_{\varepsilon_{n}}, u_{n} \equiv u_{\varepsilon_{n}}$. 
Set $v_{n}(x)=u_{n}\left(\varepsilon_{n} x+x_{n}\right), V_{n}(x)=V\left(\varepsilon_{n} x+x_{n}\right)$ and $v_{n} \rightarrow v_{0}, x_{n} \rightarrow x_{0} \in M$ as $n \rightarrow \infty$. Then, by (4-1) and (4-25) as $n \rightarrow \infty$,

$$
\begin{aligned}
\frac{1}{2} \mathscr{T}=\varepsilon_{n}^{-N} \frac{1}{2} \int_{\mathbb{R}^{N}}\left(\varepsilon_{n}^{2}\left|\nabla u_{n}\right|^{2}+\right. & \left.V(x)\left|u_{n}\right|^{2}\right) d x \\
& -\varepsilon_{n}^{-N} \frac{1}{2} \int_{B_{\varepsilon_{n} \rho_{n}}\left(x_{n}\right)}\left(\varepsilon_{n}^{2}\left|\nabla u_{n}\right|^{2}+V(x)\left|u_{n}\right|^{2}\right) d x \rightarrow 0,
\end{aligned}
$$

which is contradictory with (4-28). We have completed the proof of Lemma 4.6.

Before we treat the decay estimate of $u_{\varepsilon}$ at infinity, we need to establish more integration estimates based on Lemma 4.6.

Note that by the assumptions in $\left(H_{2}\right)$ and $\left(H_{3}\right)$, then for any fixed $p>1$, there exists a positive constant $C_{1}=C_{1}(p)$ depending on $p$ such that

$$
f(s) \leq \frac{1}{16} \max _{\xi \in \bar{\Lambda}} \frac{V(\xi)}{K(\xi)} s+C_{1}|s|^{p} .
$$

Furthermore we have a relation between $\|u\|_{\varepsilon}$ and $\int_{\Lambda} K(x)|u|^{p+1} d x$ for any $1<p<\frac{N+2}{N-2}$ as follows, which comes from Lemma 2.1 of [Yin and Zhang 2009].

Lemma 4.7. Under the assumptions $\left(H_{1}\right)$ and $\left(H_{4}\right)$, for each $\varepsilon \in(0,1]$, then there exists a positive constant $C_{2}=C_{2}(p)$ depending only on $p$ such that

$$
\int_{\Lambda} K(x)|u|^{p+1} d x \leq C_{2} \varepsilon^{-N(p-1) / 2}\|u\|_{\varepsilon}^{p+1} \quad \text { for } u \in E_{\varepsilon},
$$

where the domain $\Lambda$ is defined in the assumption $\left(H_{4}\right)$.

For later use, we introduce two fixed positive numbers $K_{0}>128$ and $c>0$ such that $c^{2} \geq 128 K_{0}^{2} /\left(d_{0}^{2} V_{1}\right)$, where $d_{0}=\operatorname{dist}(\partial \Lambda, M)>0$ and $V_{1}=\frac{1}{2} \min _{x \in \Lambda} V(x)>0$.

Set $\nu_{0}=\min \left\{d_{0} / K_{0},\left(16 C_{1} C_{2}\right)^{-2 /(p-1)}\right\}$, where $C_{1}$ and $C_{2}$ are given in (4-29)(4-30). Take $\varepsilon_{1}=\min \left\{\varepsilon_{0}\left(v_{0}\right), d_{0} /\left(K_{0} \rho_{0}\left(v_{0}\right)\right),(\ln 2) / c\right\}$, where $\varepsilon_{0}\left(v_{0}\right)$ and $\rho_{0}\left(v_{0}\right)$ are given in Lemma 4.6. From now on, we always assume $\varepsilon<\varepsilon_{1}$ and $v<v_{0}$ in (4-26)-(4-27).

It follows from (4-26) that, for $\varepsilon<\varepsilon_{1}$ and $v<v_{0}$,

$$
\operatorname{dist}\left(x_{\varepsilon}, \partial \Lambda\right)>\frac{d_{0}}{2} \quad \text { and } \quad \varepsilon \rho_{0}\left(v_{0}\right)<\frac{d_{0}}{K_{0}} .
$$

Define $\Omega_{n, \varepsilon}=\mathbb{R}^{N} \backslash B_{R_{n, \varepsilon}}\left(x_{\varepsilon}\right)$ with $R_{n, \varepsilon}=e^{c \varepsilon n}$ and let $\tilde{n}>\hat{n}$ be integers such that

$$
R_{\hat{n}-1, \varepsilon}<\frac{d_{0}}{K_{0}} \leq R_{\hat{n}, \varepsilon}, \quad R_{\tilde{n}+2, \varepsilon} \leq \frac{d_{0}}{2}<R_{\tilde{n}+3, \varepsilon} .
$$


By the second inequality in (4-31), one gets $R_{n, \varepsilon} \geq R_{\hat{n}, \varepsilon} \geq d_{0} / K_{0}>\varepsilon \rho_{0}\left(v_{0}\right)$ for $n \geq \hat{n}$ and $\varepsilon<\varepsilon_{1}$, and this also yields

$$
\Omega_{n, \varepsilon} \cap B_{\varepsilon \rho_{0}\left(\nu_{0}\right)}\left(x_{\varepsilon}\right)=\varnothing .
$$

Let $\chi_{n, \varepsilon}(x)$ be smooth cut-off functions such that $\chi_{n, \varepsilon}(x)=0$ in $B_{R_{n, \varepsilon}}\left(x_{\varepsilon}\right)$, $\chi_{n, \varepsilon}(x)=1$ in $\Omega_{n+1, \varepsilon}, 0 \leq \chi_{n, \varepsilon} \leq 1$ and $\left|\nabla \chi_{n, \varepsilon}\right| \leq 2 /\left(R_{n+1, \varepsilon}-R_{n, \varepsilon}\right)$.

Lemma 4.8. Under assumptions $\left(H_{1}\right)$ and $\left(H_{2}\right)$, if $\varepsilon<\varepsilon_{1}$ and $\hat{n} \leq n \leq \tilde{n}$, we have

$$
\int_{\mathbb{R}^{N}} A_{n, \varepsilon} d x \leq \frac{1}{2} \int_{\Omega_{n, \varepsilon}}\left(\varepsilon^{2}\left|\nabla u_{\varepsilon}\right|^{2}+V(x) u_{\varepsilon}^{2}\right) d x,
$$

where $A_{n, \varepsilon}(x)=\varepsilon^{2}\left|\nabla\left(\chi_{n, \varepsilon} u_{\varepsilon}\right)\right|^{2}+V(x)\left(\chi_{n, \varepsilon} u_{\varepsilon}\right)^{2}$.

Proof. For $\varepsilon<\varepsilon_{1}$, it follows from a straightforward computation that

$$
R_{n+1, \varepsilon}-R_{n, \varepsilon} \geq \frac{c \varepsilon R_{n+1, \varepsilon}}{2} .
$$

This yields

$$
\varepsilon^{2}\left|\nabla \chi_{n, \varepsilon}\right|^{2} \leq \frac{4 \varepsilon^{2}}{\left|R_{n+1, \varepsilon}-R_{n, \varepsilon}\right|^{2}} \leq \frac{16}{c^{2} R_{n+1, \varepsilon}^{2}} .
$$

From the choice of $c$, for $\varepsilon<\varepsilon_{1}$ and $\hat{n} \leq n \leq \tilde{n}$, we arrive at

$$
\frac{128}{c^{2} R_{n+1, \varepsilon}^{2}} \leq V(x) \text { for } x \in\left\{x: R_{n, \varepsilon} \leq\left|x-x_{\varepsilon}\right|<R_{n+1, \varepsilon}\right\} .
$$

Noting that $\nabla \chi_{n, \varepsilon}$ is supported in $\left\{x: R_{n, \varepsilon} \leq\left|x-x_{\varepsilon}\right|<R_{n+1, \varepsilon}\right\}$, then for $\varepsilon<\varepsilon_{1}$ and $\hat{n} \leq n \leq \tilde{n}$, by (4-35) and (4-36), we obtain

$$
\varepsilon^{2}\left|\nabla \chi_{n, \varepsilon}\right|^{2} \leq \frac{1}{8} V(x) \text { in } \mathbb{R}^{N} .
$$

Multiplying (2-1) by $\chi_{n, \varepsilon}^{2} u_{\varepsilon}$ and integrating over $\mathbb{R}^{N}$ yields

$$
\int_{\mathbb{R}^{N}} A_{n, \varepsilon} d x=I+I I+I I I,
$$

where

$$
\begin{aligned}
I & =\int_{\Omega_{n, \varepsilon}} \varepsilon^{2}\left|\nabla \chi_{n, \varepsilon}\right|^{2} u_{\varepsilon}^{2} d x, \\
I I & =\int_{\Lambda \cap \Omega_{n, \varepsilon}} K(x) f\left(u_{\varepsilon}\right) \chi_{n, \varepsilon}^{2} u_{\varepsilon} \\
& \leq \frac{1}{16} \int_{\Lambda \cap \Omega_{n, \varepsilon}} V(x) u_{\varepsilon}^{2} d x+C_{1} \int_{\Lambda \cap \Omega_{n, \varepsilon}} K(x)\left|u_{\varepsilon}\right|^{p+1} d x, \\
I I I & =\int_{\left(\mathbb{R}^{N} \backslash \Lambda\right) \cap \Omega_{n, \varepsilon}} g_{\varepsilon}\left(x, u_{\varepsilon}\right) \chi_{n, \varepsilon}^{2} u_{\varepsilon} d x .
\end{aligned}
$$


By (4-37), we have

$$
|I| \leq \frac{1}{8} \int_{\Omega_{n, \varepsilon}} V(x) u_{\varepsilon}^{2} d x
$$

Next we treat $|I I|$.

Clearly, we only need to consider the case $\Lambda \cap \Omega_{n, \varepsilon} \neq \varnothing$. In this situation, there is a set $\Sigma_{n, \varepsilon}$ such that $\Sigma_{n, \varepsilon} \cap \Omega_{n, \varepsilon}$ has the uniform cone property and $\Lambda \subset \Sigma_{n, \varepsilon} \subset$ $\Lambda_{r_{0}}=\left\{x: \operatorname{dist}(x, \Lambda) \leq r_{0}\right\}$, where $r_{0}>0$ is a small constant such that $V(x) \geq V_{1}$ holds true for $x \in \Lambda_{2 r_{0}}$.

By (4-30), one has

$$
\begin{aligned}
\int_{\Sigma_{n, \varepsilon} \cap \Omega_{n, \varepsilon}} K(x)\left|u_{\varepsilon}\right|^{p+1} d x & \\
& \leq C_{2} \varepsilon^{-N(p-1) / 2}\left(\int_{\Sigma_{n, \varepsilon} \cap \Omega_{n, \varepsilon}}\left(\varepsilon^{2}\left|\nabla u_{\varepsilon}\right|^{2}+V(x) u_{\varepsilon}^{2}\right) d x\right)^{(p+1) / 2} .
\end{aligned}
$$

In addition, by (4-33), we arrive at $\Sigma_{n, \varepsilon} \cap \Omega_{n, \varepsilon} \subset \mathbb{R}^{N} \backslash B_{\varepsilon \rho_{0}\left(\nu_{0}\right)}\left(x_{\varepsilon}\right)$ for $\varepsilon<\varepsilon_{1}$ and $n \geq \hat{n}$. Thus, it follows from (4-27), (4-39) and the definition of $v_{0}$ that

$$
|I I| \leq \frac{1}{8} \int_{\Omega_{n, \varepsilon}}\left(\varepsilon^{2}\left|\nabla u_{\varepsilon}\right|^{2}+V(x) u_{\varepsilon}^{2}\right) d x .
$$

Finally, we estimate $|I I I|$.

Similar to the proof of (2-3), for $\varepsilon<\varepsilon_{1}$, we have

$$
|I I I| \leq \int_{\Omega_{n, \varepsilon}} \frac{2 \varepsilon^{3}}{1+|x|^{\theta_{0}}} u_{\varepsilon}^{2} d x \leq \frac{1}{8} \int_{\Omega_{n, \varepsilon}}\left(\varepsilon^{2}\left|\nabla u_{\varepsilon}\right|^{2}+V(x) u_{\varepsilon}^{2}\right) d x .
$$

Combining (4-38), (4-40) with (4-41) yields the conclusion of Lemma 4.8.

From Lemma 4.8, repeating the same argument as in Lemma 3.3 of [Fei and Yin 2010] leads to the following result.

Lemma 4.9. Under the assumptions of Lemma 4.8, for small $\varepsilon<\varepsilon_{1}$, one has

$$
\int_{\mathbb{R}^{N}}\left|\nabla\left(\chi_{\tilde{n}, \varepsilon} u_{\varepsilon}\right)\right|^{2} d x \leq C \varepsilon^{N-2} 2^{-(\ln 2) /(c \varepsilon)} .
$$

Next, we establish an estimate of $u_{\varepsilon}(x)$ for large $|x|$.

Lemma 4.10. Under the assumptions of Lemma 4.8, for $x \in \mathbb{R}^{N}$ satisfying $\left|x-x_{\varepsilon}\right| \geq$ $d_{0} / 2$, where the meaning of $x_{\varepsilon}$ is given in Lemma 4.2, we have

$$
u_{\varepsilon}(x) \leq C 2^{-(\ln 2) /(2 c \varepsilon)} .
$$

Proof. First we assert that

$$
\max _{\bar{\Lambda}} u_{\varepsilon} \leq C,
$$


where $C>0$ is independent of small $\varepsilon$.

In fact, for any fixed $p$ with $1<p<\frac{N+2}{N-2}$, it follows from (2-1) that $v_{\varepsilon}(x)=u_{\varepsilon}(\varepsilon x)$ satisfies

(4-45) $-\triangle v_{\varepsilon}+V(\varepsilon x) v_{\varepsilon}=K(\varepsilon x) f\left(v_{\varepsilon}\right) \leq \frac{1}{16} V(\varepsilon x) v_{\varepsilon}+C(p) v_{\varepsilon}^{p} \quad$ in $\left.B_{d_{0}}\left(\varepsilon^{-1} x_{\varepsilon}\right)\right)$, where $C(p)$ is a positive constant dependent of $p$.

Define $a_{\varepsilon}(x)=\frac{15}{16} V(\varepsilon x)-C(p) v_{\varepsilon}^{p-1}$; then $v_{\varepsilon}(x)$ is a weak subsolution of the equation

$$
\left.-\triangle v_{\varepsilon}+a_{\varepsilon}(x) v_{\varepsilon}=0 \quad \text { in } B_{d_{0}}\left(\varepsilon^{-1} x_{\varepsilon}\right)\right) .
$$

By (4-3), then we obtain, for $\frac{N}{2}<q=\frac{2 N}{(p-1)(N-2)}$ and small $\varepsilon$,

$$
\left(\int_{B_{d_{0}}\left(\varepsilon^{-1} x_{\varepsilon}\right)}\left|a_{\varepsilon}\right|^{q} d x\right)^{1 / q} \leq C+C\left(\varepsilon^{-N / 2}\left\|u_{\varepsilon}\right\|_{\varepsilon}\right)^{2 N /(q(N-2))} \leq C .
$$

This, together with the weak Harnack inequality (see [Gilbarg and Trudinger 1983, p. 193]), yields that there is a positive constant $C$ depending only on the space dimension $N$ and the $L^{q}\left(B_{d_{0}}\left(\varepsilon^{-1} x_{\varepsilon}\right)\right)$ norm of $a_{\varepsilon}(x)$ such that

$$
\begin{aligned}
\max _{\bar{\Lambda}} u_{\varepsilon} & =u_{\varepsilon}\left(x_{\varepsilon}\right)=v_{\varepsilon}\left(\varepsilon^{-1} x_{\varepsilon}\right) \leq C\left(\int_{B_{d_{0}}\left(\varepsilon^{-1} x_{\varepsilon}\right)} v_{\varepsilon}^{2} d x\right)^{1 / 2} \\
& =C\left(\varepsilon^{-N} \int_{B_{\varepsilon d_{0}}\left(x_{\varepsilon}\right)} u_{\varepsilon}^{2} d x\right)^{1 / 2} \leq C \varepsilon^{-N / 2}\left\|u_{\varepsilon}\right\|_{\varepsilon} \leq C,
\end{aligned}
$$

namely, (4-44) is proved.

In addition, as in (4-45)-(4-46), one knows that $v_{\varepsilon}(x)=u_{\varepsilon}(\varepsilon x)$ is also a weak subsolution of the equation

$$
-\triangle v_{\varepsilon}+b_{\varepsilon}(x) v_{\varepsilon}=0,
$$

where $b_{\varepsilon}(x)=\frac{15}{16} V(\varepsilon x)-C(p) \chi_{\varepsilon}(x) v_{\varepsilon}^{p-1}-\left(1-\chi_{\varepsilon}(x)\right) \varepsilon^{3} /\left(1+|\varepsilon x|^{\theta_{0}}\right)$, and $\chi_{\varepsilon}$ is a characteristic function of $\Lambda^{\varepsilon}=\left\{\varepsilon^{-1} x: x \in \Lambda\right\}$. Moreover, $b_{\varepsilon}(x)$ has a uniform $L^{\infty}$ bound independent of small $\varepsilon$ by (4-44).

On the other hand, it is noted that for $x \in \mathbb{R}^{N}$ with $x \in \mathbb{R}^{N} \backslash B_{d_{0} / 2}\left(x_{\varepsilon}\right)$, then $B_{\varepsilon c d_{0}}(x) \subset \Omega_{\tilde{n}+1, \varepsilon}$ holds true for small $\varepsilon$ and a direct computation yields, for $2^{*}=2 N /(N-2)$,

$$
\begin{aligned}
\left(\int_{B_{c d_{0}}\left(\varepsilon^{-1} x\right)}\left|v_{\varepsilon}\right|^{2^{*}} d y\right)^{1 / 2^{*}} & \leq C \varepsilon^{-(N-2) / 2}\left(\int_{\mathbb{R}^{N}}\left|\nabla\left(\chi_{\tilde{n}, \varepsilon} u_{\varepsilon}\right)\right|^{2}(z) d z\right)^{1 / 2} \\
& \leq C 2^{-(\ln 2) /(2 c \varepsilon)} .
\end{aligned}
$$


Subsequently, with the aid of Harnack inequality [Gilbarg and Trudinger 1983, Theorem 8.17] and (4-48), we arrive at

$$
u_{\varepsilon}(x)=v_{\varepsilon}\left(\varepsilon^{-1} x\right) \leq C\left(\int_{B_{c d_{0}}\left(\varepsilon^{-1} x\right)}\left|v_{\varepsilon}\right|^{2^{*}} d y\right)^{1 / 2^{*}} \leq C 2^{-(\ln 2) /(2 c \varepsilon)},
$$

where $C>0$ depends only on $d_{0}, N$ and the uniform $L^{\infty}$ bound of $b_{\varepsilon}(x)$.

Since the $L^{\infty}$ norm of $b_{\varepsilon}(x)$ is uniformly bounded, the proof of Lemma 4.10 is complete.

Remark 4.1. By Lemma 4.10, for $\theta \geq 1$, there exists an $\varepsilon_{0}$ such that for $\varepsilon<\varepsilon_{0}$,

$$
\left|u_{\varepsilon}(x)\right| \leq \varepsilon^{\theta} \quad \text { for } x \in \mathbb{R}^{N} \backslash B_{d_{0} / 2}\left(x_{\varepsilon}\right) .
$$

Next, we show that the local maximum point $x_{\varepsilon}$ of $u_{\varepsilon}(x)$ in the domain $\bar{\Lambda}$ is also a maximum point of $u_{\varepsilon}(x)$ in the whole space.

Lemma 4.11. Under the assumptions of Lemma 4.8, $x_{\varepsilon}$ is the maximum point of $u_{\varepsilon}$ in $\mathbb{R}^{N}$.

Proof. Let $y_{\varepsilon}$ be the maximum point of $u_{\varepsilon}$ in $\mathbb{R}^{N}$; then $u_{\varepsilon}\left(y_{\varepsilon}\right)=\max _{\mathbb{R}^{N}} u_{\varepsilon} \geq$ $\max _{\bar{\Lambda}} u_{\varepsilon} \geq C$. According to (4-50), we have $y_{\varepsilon} \subset B_{d_{0} / 2}\left(x_{\varepsilon}\right) \subset \Lambda$ for small $\varepsilon$. Hence $y_{\varepsilon}=x_{\varepsilon}$ for small $\varepsilon$ by Lemma 4.5. Namely, the proof of Lemma 4.11 is completed.

Proof of Theorem 1.1. It follows from the assumption $\left(H_{5}\right)$ that there exist positive constants $\sigma_{0}, \theta_{0}, \theta_{1}$ and $\theta_{2}$ such that

$$
\beta<\left(\alpha-\theta_{1}\right) \sigma_{0}-\theta_{0} \quad \text { and } \quad 4+2\left(\alpha-\theta_{1}\right) \leq\left(\theta_{1}-1\right) \theta_{2},
$$

where $N-\frac{9}{4}<\sigma_{0}<N-2, \theta_{0}>2, \theta_{1}>1$.

We define the comparison function

$$
U(x)=\frac{1}{\left|x-x_{\varepsilon}\right|^{\sigma_{0}}} \quad \text { for } x \in \mathbb{R}^{N} \backslash B_{d_{0} / 2}\left(x_{\varepsilon}\right) .
$$

It is easy to know that $Z(x)=U(x)-\varepsilon^{2} u_{\varepsilon}(x) \geq 0$ on $\partial\left(B_{d_{0} / 2}\left(x_{\varepsilon}\right)\right)$ for small $\varepsilon$. Recalling that $v_{\varepsilon}(x)=u_{\varepsilon}(\varepsilon x)$ vanishes at infinity, this is also true for $Z(x)$.

On the other hand, using the expression for $h_{\varepsilon}\left(x, u_{\varepsilon}\right)$ and noting that $\sigma_{0}<N-2$, we conclude from (4-50) that $\Delta Z=\Delta U-\varepsilon^{2} \Delta u_{\varepsilon} \leq 0$ holds for $x \in \mathbb{R}^{N} \backslash B_{d_{0} / 2}\left(x_{\varepsilon}\right)$ and sufficiently small $\varepsilon$.

Thus, by the maximum principle, we deduce $u_{\varepsilon} \leq U / \varepsilon^{2}$ in $x \in \mathbb{R}^{N} \backslash B_{d_{0} / 2}\left(x_{\varepsilon}\right)$. This and the uniform boundedness of $x_{\varepsilon}$ imply

$$
u_{\varepsilon}(x) \leq \frac{C}{\varepsilon^{2}\left(1+|x|^{\sigma_{0}}\right)} \quad \text { in } \mathbb{R}^{N} \backslash \Lambda .
$$


Next we verify that $u_{\varepsilon}$ actually solves Equation (1-1). Indeed, since $f(s)=O\left(s^{\alpha}\right)$ near $s=0$, together with (4-50) we have, for small $\varepsilon$,

$$
f\left(u_{\varepsilon}\right) \leq C\left|u_{\varepsilon}\right|^{\alpha} \quad \text { in } \mathbb{R}^{N} \backslash \Lambda .
$$

Combining (4-50)-(4-53), we have, for small $\varepsilon$,

$$
K(x) f\left(u_{\varepsilon}\right) \leq C k\left(1+|x|^{\beta}\right)\left|u_{\varepsilon}\right|^{\alpha} \leq \frac{\varepsilon^{3}}{1+|x|^{\theta_{0}}}\left|u_{\varepsilon}\right| \quad \text { in } \mathbb{R}^{N} \backslash \Lambda .
$$

Choose two positive numbers $\theta_{3}$ and $\theta_{4}$ such that

$$
\beta<\left(\alpha-\theta_{3}\right) \sigma_{0}-N \text { and } 2+2\left(\alpha-\theta_{3}\right) \leq \theta_{3} \theta_{4} .
$$

Collecting (4-50), (4-52), (4-53), and (4-55) yields for small $\varepsilon$,

$$
K(x) f\left(u_{\varepsilon}\right) \leq C k\left(1+|x|^{\beta}\right)\left|u_{\varepsilon}\right|^{\alpha-\theta_{3}}\left|u_{\varepsilon}\right|^{\theta_{3}} \leq \frac{\varepsilon}{1+|x|^{N}} \quad \text { in } \mathbb{R}^{N} \backslash \Lambda .
$$

Therefore, it follows from (4-54) and (4-56) that $g_{\varepsilon}\left(x, u_{\varepsilon}\right) \equiv K(x) f\left(u_{\varepsilon}\right)$ holds true in $\mathbb{R}^{N} \backslash \Lambda$ and subsequently $u_{\varepsilon}$ solves the original equation (1-1). In addition, noting that $N-\frac{9}{4}<\sigma_{0}$, then the estimate (4-52) leads to $u_{\varepsilon} \in L^{2}\left(\mathbb{R}^{N}\right)$ for $N \geq 5$.

Finally, combining the conclusions in Lemma 4.2, Lemma 4.5 and Lemma 4.11, in order to finish the proof of Theorem 1.1, we only need to verify (1-12). Set $M=\left\{x_{0}\right\}$, due to (4-26), one has $x_{\varepsilon} \rightarrow x_{0}$ as $\varepsilon \rightarrow 0$. Let $v_{\varepsilon}(x)=u_{\varepsilon}\left(\varepsilon x+x_{\varepsilon}\right)$, then $v_{\varepsilon}$ is uniformly bounded in $H_{\mathrm{loc}}^{1}\left(\mathbb{R}^{N}\right)$ and satisfies the equation

$$
-\triangle v_{\varepsilon}+V\left(\varepsilon x+x_{\varepsilon}\right) v_{\varepsilon}=K\left(\varepsilon x+x_{\varepsilon}\right) f\left(v_{\varepsilon}\right), \quad x \in \mathbb{R}^{N} .
$$

As in the arguments of Lemma 4.3 or Lemma 4.5, we can show that $v_{\varepsilon}$ converges to $v \in C^{2}\left(\mathbb{R}^{N}\right) \cap H^{1}\left(\mathbb{R}^{N}\right)$ in $C_{\text {loc }}^{2}\left(\mathbb{R}^{N}\right)$ as $\varepsilon \rightarrow 0$. With the aid of (4-43), $v_{\varepsilon}$ converges to $v$ in $L^{\infty}\left(\mathbb{R}^{N}\right)$ as $\varepsilon \rightarrow 0$. Therefore $v$ is a solution of the equation

$$
-\triangle v+V\left(x_{0}\right) v=K\left(x_{0}\right) f(v), \quad x \in \mathbb{R}^{N}
$$

moreover, by virtue of strong maximum principle, $v>0$ can be derived. On the other hand, as a consequence of Theorem 2 [Gidas et al. 1981] and the subsequent remark, $v$ is radially symmetric and decays exponentially.

Thus the proof of Theorem 1.1 is completed.

\section{References}

[Ambrosetti and Malchiodi 2007] A. Ambrosetti and A. Malchiodi, "Concentration phenomena for nonlinear Schrödinger equations: recent results and new perspectives", pp. 19-30 in Perspectives in nonlinear partial differential equations, edited by $\mathrm{H}$. Berestycki et al., Contemp. Math. 446, Amer. Math. Soc., Providence, RI, 2007. MR 2008j:35037 Zbl 1200.35106 
[Ambrosetti and Wang 2005] A. Ambrosetti and Z.-Q. Wang, "Nonlinear Schrödinger equations with vanishing and decaying potentials", Differential Integral Equations 18:12 (2005), 1321-1332. MR 2006k:35071 Zbl 1210.35087

[Ambrosetti et al. 2005] A. Ambrosetti, V. Felli, and A. Malchiodi, "Ground states of nonlinear Schrödinger equations with potentials vanishing at infinity”, J. Eur. Math. Soc. 7:1 (2005), 117-144. MR 2006f:35049 Zbl 1064.35175

[Berestycki and Lions 1983] H. Berestycki and P.-L. Lions, "Nonlinear scalar field equations, I: Existence of a ground state", Arch. Rational Mech. Anal. 82:4 (1983), 313-345. MR 84h:35054a Zbl 0533.35029

[Bonheure and Van Schaftingen 2008] D. Bonheure and J. Van Schaftingen, "Bound state solutions for a class of nonlinear Schrödinger equations", Rev. Mat. Iberoam. 24:1 (2008), 297-351. MR 2009d:35069 Zbl 1156.35084

[Byeon and Wang 2006] J. Byeon and Z.-Q. Wang, "Spherical semiclassical states of a critical frequency for Schrödinger equations with decaying potentials", J. Eur. Math. Soc. 8:2 (2006), 217-228. MR 2007e:35073 Zbl 1245.35036

[Costa and Tehrani 2001] D. G. Costa and H. Tehrani, "On a class of asymptotically linear elliptic problems in $\mathbb{R}^{N}$ ", J. Differential Equations 173:2 (2001), 470-494. MR 2002g:35065 Zbl 1098.35526

[Dávila et al. 2007] J. Dávila, M. del Pino, M. Musso, and J. Wei, "Standing waves for supercritical nonlinear Schrödinger equations”, J. Differential Equations 236:1 (2007), 164-198. MR 2009b:35389 Zbl 1124.35082

[Ekeland 1990] I. Ekeland, Convexity methods in Hamiltonian mechanics, Ergebnisse der Mathematik und ihrer Grenzgebiete, III 19, Springer, Berlin, 1990. MR 91f:58027 Zbl 0707.70003

[Fei and Yin 2010] M. Fei and H. Yin, "Existence and concentration of bound states of nonlinear Schrödinger equations with compactly supported and competing potentials", Pacific J. Math. 244:2 (2010), 261-296. MR 2011j:35082 Zbl 1189.35304

[Gidas et al. 1981] B. Gidas, W. M. Ni, and L. Nirenberg, "Symmetry of positive solutions of nonlinear elliptic equations in $\mathbf{R}^{n}$ ", pp. 369-402 in Mathematical analysis and applications, Part A, edited by L. Nachbin, Adv. in Math. Suppl. Stud. 7, Academic, New York, 1981. MR 84a:35083 Zbl 0469.35052

[Gilbarg and Trudinger 1983] D. Gilbarg and N. S. Trudinger, Elliptic partial differential equations of second order, 2nd ed., Grundlehren der Mathematischen Wissenschaften 224, Springer, Berlin, 1983. MR 86c:35035 Zbl 0562.35001

[Gui 1996] C. Gui, "Existence of multi-bump solutions for nonlinear Schrödinger equations via variational method”, Comm. Partial Differential Equations 21:5-6 (1996), 787-820. MR 98a:35122 Zbl 0857.35116

[Jeanjean and Tanaka 2002] L. Jeanjean and K. Tanaka, "A positive solution for an asymptotically linear elliptic problem on $\mathbb{R}^{N}$ autonomous at infinity", ESAIM Control Optim. Calc. Var. 7 (2002), 597-614. MR 2003f:35113 Zbl 1225.35088

[Liu and Wang 2004] Z. Liu and Z.-Q. Wang, "Existence of a positive solution of an elliptic equation on $\mathbb{R}^{N}$ ", Proc. Roy. Soc. Edinburgh Sect. A 134:1 (2004), 191-200. MR 2005c:35101 Zbl 1067.35029

[Liu et al. 2006] Z. Liu, J. Su, and T. Weth, "Compactness results for Schrödinger equations with asymptotically linear terms", J. Differential Equations 231:2 (2006), 501-512. MR 2009d:35095 Zbl 05115328 
[Liu et al. 2008] C. Liu, Z. Wang, and H.-S. Zhou, "Asymptotically linear Schrödinger equation with potential vanishing at infinity”, J. Differential Equations 245:1 (2008), 201-222. MR 2009h:35144 Zbl 1188.35181

[del Pino and Felmer 1996] M. del Pino and P. L. Felmer, "Local mountain passes for semilinear elliptic problems in unbounded domains", Calc. Var. Partial Differential Equations 4:2 (1996), 121-137. MR 97c:35057 Zbl 0844.35032

[del Pino et al. 2007] M. del Pino, M. Kowalczyk, and J.-C. Wei, "Concentration on curves for nonlinear Schrödinger equations”, Comm. Pure Appl. Math. 60:1 (2007), 113-146. MR 2007h:35113 Zbl 1123.35003

[Rabinowitz 1992] P. H. Rabinowitz, "On a class of nonlinear Schrödinger equations", Z. Angew. Math. Phys. 43:2 (1992), 270-291. MR 93h:35194 Zbl 0763.35087

[Stuart and Zhou 1999] C. A. Stuart and H. S. Zhou, "Applying the mountain pass theorem to an asymptotically linear elliptic equation on $\mathbf{R}^{N}$ ", Comm. Partial Differential Equations 24:9-10 (1999), 1731-1758. MR 2000g:35052 Zbl 0935.35043

[Wang and Zeng 1997] X. Wang and B. Zeng, "On concentration of positive bound states of nonlinear Schrödinger equations with competing potential functions”, SIAM J. Math. Anal. 28:3 (1997), 633-655. MR 98e:81032 Zbl 0879.35053

[Willem 1996] M. Willem, Minimax theorems, Progress in Nonlinear Differential Equations and their Applications 24, Birkhäuser, Boston, MA, 1996. MR 97h:58037 Zbl 0856.49001

[Yin and Zhang 2009] H. Yin and P. Zhang, "Bound states of nonlinear Schrödinger equations with potentials tending to zero at infinity", J. Differential Equations 247:2 (2009), 618-647. MR 2010m:35169 Zbl 1178.35353

Received August 25, 2011. Revised August 17, 2012.

\section{MingWEN FEI}

DEPARTMENT OF MATHEMATICS \& IMS

NANJING UNIVERSITY

210093 NANJING

CHINA

and

ACADEmy of Mathematics And Systems SCIEnCE

Chinese ACADEMY of SCIENCES

100190 BEIJING

CHINA

mwfei@amss.ac.cn

HUICHENG YIN

DEPARTMENT OF MATHEMATICS \& IMS

NANJING UNIVERSITY

210093 NANJING

CHINA

huicheng@nju.edu.cn 


\title{
PACIFIC JOURNAL OF MATHEMATICS
}

\author{
msp.org/pjm
}

Founded in 1951 by E. F. Beckenbach (1906-1982) and F. Wolf (1904-1989)

\section{EDITORS}

V. S. Varadarajan (Managing Editor)

Department of Mathematics

University of California

Los Angeles, CA 90095-1555

pacific@math.ucla.edu

Paul Balmer

Department of Mathematics

University of California

Los Angeles, CA 90095-1555

balmer@math.ucla.edu

Daryl Cooper

Department of Mathematics

University of California

Santa Barbara, CA 93106-3080 cooper@math.ucsb.edu

Jiang-Hua $\mathrm{Lu}$

Department of Mathematics

The University of Hong Kong

Pokfulam Rd., Hong Kong jhlu@maths.hku.hk
Don Blasius

Department of Mathematics University of California

Los Angeles, CA 90095-1555

blasius@math.ucla.edu

Robert Finn

Department of Mathematics Stanford University

Stanford, CA 94305-2125

finn@math.stanford.edu

Sorin Popa

Department of Mathematics

University of California

Los Angeles, CA 90095-1555

popa@math.ucla.edu

Paul Yang

Department of Mathematics

Princeton University

Princeton NJ 08544-1000

yang@math.princeton.edu

\section{PRODUCTION}

Silvio Levy, Scientific Editor, production@msp.org

\section{SUPPORTING INSTITUTIONS}

ACADEMIA SINICA, TAIPEI

CALIFORNIA INST. OF TECHNOLOGY

INST. DE MATEMÁTICA PURA E APLICADA

KEIO UNIVERSITY

MATH. SCIENCES RESEARCH INSTITUTE

NEW MEXICO STATE UNIV.

OREGON STATE UNIV.

\author{
STANFORD UNIVERSITY \\ UNIV. OF BRITISH COLUMBIA \\ UNIV. OF CALIFORNIA, BERKELEY \\ UNIV. OF CALIFORNIA, DAVIS \\ UNIV. OF CALIFORNIA, LOS ANGELES \\ UNIV. OF CALIFORNIA, RIVERSIDE \\ UNIV. OF CALIFORNIA, SAN DIEGO \\ UNIV. OF CALIF., SANTA BARBARA
}

\author{
Vyjayanthi Chari \\ Department of Mathematics \\ University of California \\ Riverside, CA 92521-0135 \\ chari@math.ucr.edu \\ Kefeng Liu \\ Department of Mathematics \\ University of California \\ Los Angeles, CA 90095-1555 \\ liu@math.ucla.edu \\ Jie Qing \\ Department of Mathematics \\ University of California \\ Santa Cruz, CA 95064 \\ qing@cats.ucsc.edu
}

These supporting institutions contribute to the cost of publication of this Journal, but they are not owners or publishers and have no responsibility for its contents or policies.

See inside back cover or msp.org/pjm for submission instructions.

The subscription price for 2013 is US \$400/year for the electronic version, and \$485/year for print and electronic.

Subscriptions, requests for back issues and changes of subscribers address should be sent to Pacific Journal of Mathematics, P.O. Box 4163, Berkeley, CA 94704-0163, U.S.A. The Pacific Journal of Mathematics is indexed by Mathematical Reviews, Zentralblatt MATH, PASCAL CNRS Index, Referativnyi Zhurnal, Current Mathematical Publications and the Science Citation Index.

The Pacific Journal of Mathematics (ISSN 0030-8730) at the University of California, c/o Department of Mathematics, 798 Evans Hall \#3840, Berkeley, CA 94720-3840, is published monthly except July and August. Periodical rate postage paid at Berkeley, CA 94704, and additional mailing offices. POSTMASTER: send address changes to Pacific Journal of Mathematics, P.O. Box 4163, Berkeley, CA 94704-0163.

PJM peer review and production are managed by EditFLOW ${ }^{\circledR}$ from Mathematical Sciences Publishers.

PUBLISHED BY

mathematical sciences publishers

nonprofit scientific publishing

http://msp.org/

(C) 2013 Mathematical Sciences Publishers 


\section{PACIFIC JOURNAL OF MATHEMATICS}

Volume $261 \quad$ No. $2 \quad$ February 2013

Geography of simply connected nonspin symplectic 4-manifolds with positive signature

Anar Akhmedov, Mark C. Hughes and B. Doug Park

Schur-Horn theorems in $\mathrm{II}_{\infty}$-factors

283

MARTÍN ARGERAMI and PEDRo MASSEY

Classification of positive solutions for an elliptic system with a higher-order

fractional Laplacian

JiNGBo DOU and CHANGZHENG QU

Bound states of asymptotically linear Schrödinger equations with compactly supported potentials

MingWEN FEI and HUICHENG YiN

Type I almost homogeneous manifolds of cohomogeneity one, III

DANIEL GUAN

The subrepresentation theorem for automorphic representations

MARCELA HANZER

Variational characterizations of the total scalar curvature and eigenvalues of the

Laplacian

Seungsu Hwang, JeOngwook Chang and GabJin Yun

Fill-ins of nonnegative scalar curvature, static metrics, and quasi-local mass

JEFFREY L. JAUREGUI

Operator algebras and conjugacy problem for the pseudo-Anosov automorphisms of a surface

\section{IGOR NIKOLAEV}

Connected sums of closed Riemannian manifolds and fourth-order conformal invariants

DAVID RASKE

Ruled minimal surfaces in the three-dimensional Heisenberg group

Heayong Shin, Young Wook Kim, Sung-Eun Koh, Hyung Yong LeE and SEONG-DEOG YANG

$G$-bundles over elliptic curves for non-simply laced Lie groups and configurations of lines in rational surfaces

MANG XU and JIAJIN ZHANG 